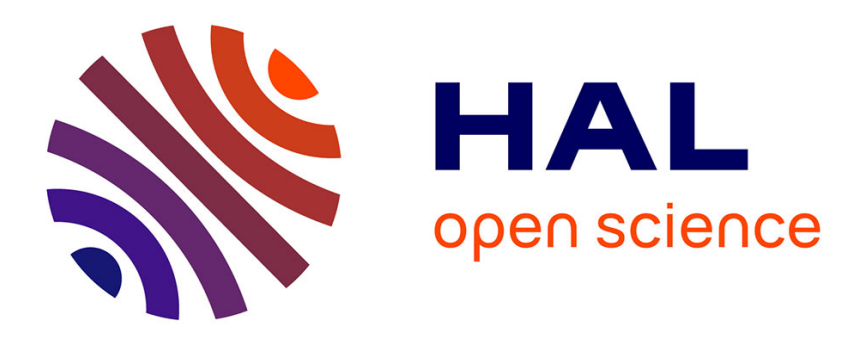

\title{
An Evolving Switching Surface Model for Ferromagnetic Hysteresis
}

D. Mukherjee, K. Danas

\section{To cite this version:}

D. Mukherjee, K. Danas. An Evolving Switching Surface Model for Ferromagnetic Hysteresis. Journal of Applied Physics, 2019, 125 (3), pp.033902. 10.1063/1.5051483 . hal-02394300

\section{HAL Id: hal-02394300 \\ https://hal.science/hal-02394300}

Submitted on 4 Dec 2019

HAL is a multi-disciplinary open access archive for the deposit and dissemination of scientific research documents, whether they are published or not. The documents may come from teaching and research institutions in France or abroad, or from public or private research centers.
L'archive ouverte pluridisciplinaire HAL, est destinée au dépôt et à la diffusion de documents scientifiques de niveau recherche, publiés ou non, émanant des établissements d'enseignement et de recherche français ou étrangers, des laboratoires publics ou privés. 


\title{
An Evolving Switching Surface Model for Ferromagnetic Hysteresis
}

\author{
D. Mukherjee ${ }^{1}$ and K. Danas ${ }^{1, a)}$ \\ LMS, C.N.R.S, École Polytechnique, Palaiseau, 91128, France
}

(Dated: 22 November 2019)

\begin{abstract}
We propose a thermodynamically consistent rate-independent three-dimensional model of magnetic hysteresis in terms of energetic and dissipation potentials making use of a relatively small number of model parameters that is capable of being implemented in a general incremental numerical setting. The dissipation process occurring during magnetization/demagnetization is described by a power-law potential, which leads to rateindependence at a certain limit of the rate-dependent exponent. The incorporation of isotropic hardening in the model enables us to describe phenomenologically at the macroscopic scale both nucleation and pinning type constitutive responses. We further model the symmetric and asymmetric minor loops by employing the idea of a bounding surface, which was originally introduced in the context of mechanical plasticity. Our model shows a very good agreement with experiments for spark plasma sintered NdFeB magnets, where nucleation is found to be the primary mechanism of coercivity. We also use our model to probe experiments for melt-spun $\mathrm{NdFeB}$ ribbons and powders, where both nucleation and pinning mechanisms are experimentally found to be significant. Moreover, we correlate the proposed model parameters with the underlying mechanisms for coercivity. Finally, we probe the predictive capability of the proposed model by first fitting an experimental minor loop, and then use it to successfully predict the remaining minor loops, obtained from that experiment. We also construct a FORC diagram for the floppy disc material and compare it with the corresponding experimental data.
\end{abstract}

Keywords: Thermodynamic potentials, Ferromagnetic switching, Bounding surface

\section{INTRODUCTION}

Recent developments in the manufacturing of rare earth (RE) metal based polycrystalline permanent magnets $^{1-6}$ and recent experiments with $\mathrm{RE}$ magnetic particle filled magnetorheological elastomers (MREs) ${ }^{7-10}$ open a vast window of opportunity for designing different microstructures to achieve specific magnetic properties at the macroscale. Theoretical modeling of the macroscopic behavior of metallic magnets/MREs requires efficient constitutive models, which must be thermodynamically consistent at the same time. In this regard, $\mathrm{Hu}-$ ber ${ }^{11}$ proposed such a model for ferroelectric materials that exhibits hysteresis, in terms of an energetic potential and a switching surface, which defines the boundary of the energetic/switching response. This switching surface model has been modified later by Cocks and Mcmeeking ${ }^{12}$, McMeeking and Landis ${ }^{13}$, Landis ${ }^{14}$ and Klinkel $^{15}$ and has been used extensively in finite element computations of dissipative electro/magneto mechanical materials $^{16,17}$. The switching surface idea can be directly related to the coercivity of a magnet, which serves to describe the effect of complex domain wall motion and interaction at the macroscale.

Specifically, coercivity constitutes one of the most important dissipative mechanisms in permanent magnets. The magnetic field, that is required to demagnetize a magnet completely from a fully magnetized (saturated) state is the coercive field, which is, in turn, the measure of coercivity. Experiments show two different kinds

\footnotetext{
a)Electronic mail: konstantinos.danas@polytechnique.edu
}

of coercivity mechanisms in hard magnets; (a) magnetic domain nucleation and (b) magnetic domain pinning. Most of the hard magnets in nature are found to magnetize/demagnetize through a combined effect of domain nucleation and domain wall pinning. The type of a magnet is determined based on the mechanism that is dominant. For example, in sintered RE magnets like NdFeB, nucleation is found to be dominant ${ }^{18-22}$, whereas, in most of the steels like Si steel, MnMg steel, the coercivity arises due to domain wall pinning ${ }^{23}$. Recent experiments by Li et al. ${ }^{24}$ have shown that both nucleation and pinning mechanisms play equally important roles in the magnetization/demagnetization of melt-spun $\mathrm{NdFeB}$ ribbons and powders.

Magnetic domain nucleation is a phenomena where reverse magnetic domains nucleate near the crystal defects. This mechanism ensures high susceptibility at the beginning of magnetization and consequently, magnetic saturation is achieved at a lower magnetic field ${ }^{18-20}$. Experiments by Huang et al. ${ }^{6}$ confirm the nucleation mechanism in sintered $\mathrm{NdFeB}$ magnets by showing that virgin state sintered $\mathrm{NdFeB}$ magnets do exhibit high susceptibility at the beginning. Kronmüller, Durst, and Sagawa ${ }^{18}$ show that the micromagnetic domain properties and temperature dependence of the coercive field in sintered $\mathrm{NdFeB}$ magnets are direct consequences of domain nucleation.

In turn, most of the traditional ferromagnetic materials like steel and Fe-Ni-Co alloys exhibit a coercivity mechanism known as domain wall pinning ${ }^{25}$. In this case, the magnetic domains remain strongly pinned to the crystal grain boundaries, and as a result the initial magnetic susceptibility during magnetization remains low. At the onset of switching, the domain walls get unpinned 
from the defects and consequently, reversed domains rotate quickly towards the direction of the magnetic field, causing a rapid increase in the magnetization, termed as magnetic switching ${ }^{16,17}$. Notably, for pinning-type magnets the switching starts after the local magnetic field reaches a threshold, whereas, for nucleation-type magnets, the switching starts even under a very small local field. This key idea of early initiation of the magnetic switching plays a pivotal role in defining the evolution of the magnetic switching surface, which is investigated in detail in this paper.

During the initial development of macroscopic constitutive models of ferromagnetic hysteresis, most of the magnets were made of steel. Thus, early experiments with steels ${ }^{23}$ mostly show a pinning-type initial magnetization response. Hence, classical hysteresis models like the Preisach ${ }^{26}$ and Jiles and Atherton ${ }^{27}$ models are based on the domain wall pinning theory. Moreover, it is very difficult to establish thermodynamic consistency of these models in complex non-uniaxial loading histories. In view of this, one can categorize the thermodynamically consistent models for ferro-electric/magnetic hysteresis under two broad distinct classes. The first class $^{28-31}$ considers a double well energetic potential associated with a ferro-electric/magnetic single crystal. Such models allow the single crystals to switch between two distinct states of polarization/magnetization (similar to a phase transition). Various homogenization ${ }^{32-34}$ and statistical averaging ${ }^{31,35,36}$ techniques have been employed to extend such single-crystal switching laws to model polycrystalline ferro-electrics/magnets. In those models, it is very difficult to obtain intermediate polarization/magnetization in-between the two extremities of the coersive response. Nevertheless, experiments show that the ferro-electric/magnetic single crystals and especially polycrystals do exhibit such intermediate polarization/magnetization states ${ }^{37,38}$. Thus, a second class of more phenomenological models ${ }^{11,12}$ have been proposed. The latter are based on a convex energetic potential and a switching surface (similar to yield surface in mechanical plasticity), that dictates the dissipative behavior of the single crystals. Extension of such single crystal plasticity models to polycrystalline materials is typically obtained either through homogenization ${ }^{37}$ or by proposing constitutive laws based on a convex potential and a switching surface, directly at the macro scale ${ }^{13-16}$.

Even more interestingly, complex symmetric and asymmetric minor loops are also observed in a magnet either due to fully reversed loading cycles with amplitude less than the coercive field, or due to partially reversed cycles. The minor loops are typically modeled by introducing a set of history-dependent parameters in the classical Jiles and Atherton ${ }^{27}$ model $^{39,40}$. However, those models are not based on energetic and dissipation potentials and thus their thermodynamic consistency (and positive magnetic dissipation) is extremely difficult to be addressed for all possible loadings, including cyclic ones. On the other hand, the double-well potential-based
Bergqvist ${ }^{31}$ model and its successors ${ }^{35,36,41,42}$ trace the minor loops, but again, no clear connection between the minor loops and the loading history is established by these models. In turn, the existing macroscopic switching surface models ${ }^{13-16}$ have only been used so far to model the outer coersive response but have not been extended to accurately model such minor loops. Such minor loops and incomplete magnetized responses are expected to be extremely important in recently fabricated $\mathrm{NdFeB}$ particle-filled elastomers ${ }^{42}$, which constitute novel materials with great potential technological applications in sensors and actuators, as well as in haptic devices.

In view of this, following the works of Landis ${ }^{14}$ and Klinkel ${ }^{15}$, we propose here a thermodynamically consistent model by use of frame-invariant free energy functions and dissipation potentials, thus ensuring positive work during any loading history. In addition, we model the minor loops by evolving the switching surface as a function of a set of history-dependent state variables. Those variables mimic (mechanical) plasticity and specifically the notion of the bounding surface proposed by Dafalias and Popov $^{43}$ (see also ${ }^{44,45}$ ). Following this idea, Chaboche, Van, and Cordier ${ }^{46}$ proposed a bounding surface that lies in the plastic strain space (see comprehensive review ${ }^{47}$ ). In this work, we extend the latter key idea in the context of ferromagnetism by introducing internal variables such as the accumulated remanent $h$-field, as well as a number of proximity variables serving to measure and memorize the distance of the actual $h$-field from the outer bounding surface. The complete set of variables is employed to model the initial magnetization, the fully magnetized coersive loop and ultimately symmetric and asymmetric first order minor loops in magnetic polycrystalline materials. In the case of no initial magnetization and no minor loops, the present model fully recovers the models of Landis ${ }^{14}$ and Klinkel ${ }^{15}$.

We organize this article as follows. In Section II we recall the fundamental governing Maxwell's equations for magnetism and define the relevant variables of the problem. In Section III, we develop a thermodynamically consistent constitutive model with hysteresis by defining the general thermodynamic framework for dissipative systems. In Section III A, we propose a hardening function during magnetic switching and then define an appropriate isotropic hardening function in Section IIIB. The idea of a bounding surface in the remanent $h$-field space is developed in Section IV A. In Section IV B, we introduce specific modifications in the evolution law of the switching surface. A detailed account of the identification of model parameters are given in Section V In Section VI, we carry out an extensive assessment of the proposed model, by probing available hysteresis data for RE-based NdFeB magnets, Co alloys, and different kind of steels. Finally, we summarize our work and draw the conclusions in Section VII. 


\section{PRELIMINARY DEFINITIONS}

We consider a static and non-deformable magnetic solid occupying a volume $\Omega_{0}$ in the three dimensional Euclidean space. In the absence of mechanical deformations, electric fields and free charges, the local magnetic field $\mathbf{b}$ and the local $h$-field $\mathbf{h}$ in $\Omega_{0}$ are governed by the following field equations

$$
\operatorname{div} \mathbf{b}=0, \quad \operatorname{curl} \mathbf{h}=0,
$$

respectively. One can then define the magnetization vector per unit current volume $\mathbf{m}$ in terms of $\mathbf{b}$ and $\mathbf{h}$ by

$$
\mathbf{b}=\mu_{0}(\mathbf{h}+\mathbf{m}) \text {. }
$$

Here, $\mu_{0}$ is the magnetic permeability in vacuum. The curl-free property of the $h$-field in $\Omega_{0}$ allows us to express $\mathbf{h}$ as the gradient of a scalar potential $\varphi$, i.e. $\mathbf{h}=-\nabla \varphi$. Similarly, the divergence-free $\mathbf{b}$ may be expressed as the curl of a vector potential $\boldsymbol{\alpha}$, i.e., $\mathbf{b}=$ curl $\boldsymbol{\alpha}$.

The magnetic response of a solid may be modeled by using either $\mathbf{b}$ or $\mathbf{h}$ (together with $\mathbf{m}$ ) as the primary variable. Some of the existing models use either $\mathbf{b}^{48-51}$, or $\mathbf{m}$ and $\mathbf{b}^{42,52}$ as the primary variable. However, from the computational point of view, the choice of the curl-free $h$-field as the primary variable has the advantage of requiring only a scalar potential field $\varphi$. As a result, most of the existing computational frameworks for magnetic solids have been developed in the $\mathbf{h}$ framework ${ }^{16,17,53}$. In the present work, we also use $\mathbf{h}$ as the primary variable in our model to ensure an efficient computational framework. The dependent variable $\mathbf{b}$ is then obtained as a function of $\mathbf{h}$ by using the proposed magnetic constitutive law. The magnetization may then be computed from (2).

\section{THE CONSTITUTIVE MODEL FOR SYMMETRIC CYCLIC LOADING}

Air and other magnetically inert media with no magnetization, are typically modeled by a linear constitutive relation $\mathbf{b}=\mu_{0} \mathbf{h}$. The constitutive relation for magnetically soft iron with no significant hysteresis is typically given by an inverse sigmoid function like the inverse Langevin function or the inverse hyperbolic tangent function or any combination of them ${ }^{51}$. In turn, the constitutive modeling of hard magnets that dissipate energy via hysteresis is not straightforward. To this end, we split $\mathbf{h}$ into an energetic part $\mathbf{h}^{e}$ and a dissipative part $\mathbf{h}^{r}$, called the remanent $h$-field, such that

$$
\mathbf{h}=\mathbf{h}^{e}+\mathbf{h}^{r} \text {. }
$$

The magnetic response is then described in terms of a Helmholtz free energy $\psi$. Following McMeeking and Landis $^{13}$ and Klinkel ${ }^{15}$, we propose an additive decomposition of the Helmholtz free energy into energetic and remanent parts, such that

$$
\rho_{0} \psi\left(\mathbf{h}, \mathbf{h}^{r}\right)=\rho_{0} \psi^{e}\left(\mathbf{h}, \mathbf{h}^{r}\right)+\rho_{0} \psi^{r}\left(\mathbf{h}^{r}\right),
$$

where $\rho_{0}$ is the reference density of the material. In the above expression, $\psi^{e}$ is the free energy associated with the energetic response, whereas $\psi^{r}$ is associated to the remanent $\mathbf{h}^{r}$. Specifically, the energetic response of the magnet is taken to be quadratic in $\mathbf{h}^{e}=\left(\mathbf{h}-\mathbf{h}^{r}\right)$, such that

$$
\rho_{0} \psi^{e}=-\frac{1}{2}\left(\mathbf{h}-\mathbf{h}^{r}\right) \cdot \boldsymbol{\mu} \cdot\left(\mathbf{h}-\mathbf{h}^{r}\right) .
$$

In this expression, $\boldsymbol{\mu}$ denotes the permeability tensor, which becomes $\mu \mathbf{I}$ for isotropic magnets (with $\mathbf{I}$ denoting the second order identity tensor). The magnetic field $\mathbf{b}$ is then obtained from the constitutive relation as

$$
\mathbf{b}=-\rho_{0} \frac{\partial \psi}{\partial \mathbf{h}}=-\rho_{0} \frac{\partial \psi^{e}}{\partial \mathbf{h}}=\boldsymbol{\mu} \cdot\left(\mathbf{h}-\mathbf{h}^{r}\right) .
$$

Furthermore, the back magnetic field $\mathbf{b}^{b}$ (in analogy to back stress in mechanics) is defined as

$$
\mathbf{b}^{b}=-\rho_{0} \frac{\partial \psi^{r}}{\partial \mathbf{h}^{r}}
$$

We note at this point that Landis ${ }^{14}$ also came up with a similar term called the back electric field in the context of ferroelastic ceramics. The back field is typically taken to be an inverse sigmoid function of $\mathbf{h}^{r 14,15}$. The specific choice of $\psi^{r}$ depends on the phenomenology of the magnet and is discussed later in Section III A.

From the second law of thermodynamics, we obtain the dissipation inequality for an isothermal, non-deforming magnetic solid as ${ }^{13-15}$

$$
\mathfrak{D}:=-\rho_{0} \dot{\psi}-\mathbf{b} \cdot \dot{\mathbf{h}} \geq 0,
$$

where $\left({ }^{\cdot}\right)$ denotes the material time derivative and $\mathfrak{D}$ is the dissipative power ${ }^{11}$. Expanding $\dot{\psi}$ in (8) yields

$$
\mathfrak{D}=\left[-\rho_{0} \frac{\partial \psi}{\partial \mathbf{h}}-\mathbf{b}\right] \cdot \dot{\mathbf{h}}+\left[-\rho_{0} \frac{\partial \psi}{\partial \mathbf{h}^{r}}\right] \cdot \dot{\mathbf{h}}^{r} \geq 0 .
$$

Substituting (6) to the above leads to

$$
\mathfrak{D}=-\rho_{0} \frac{\partial \psi}{\partial \mathbf{h}^{r}} \cdot \dot{\mathbf{h}}^{r}=\mathbf{b}^{r} \cdot \dot{\mathbf{h}}^{r} \geq 0
$$

Here, we have introduced the notion of the remanent magnetic field, $\mathbf{b}^{r}$, which is the work conjugate of $\dot{\mathbf{h}}^{r}$. The former can be written in terms of the actual magnetic field $\mathbf{b}$ minus the back magnetic field $\mathbf{b}^{b}$, i.e.,

$$
\mathbf{b}^{r}=-\rho_{0} \frac{\partial \psi}{\partial \mathbf{h}^{r}}=-\mathbf{b}-\rho_{0} \frac{\partial \psi^{r}}{\partial \mathbf{h}^{r}}=-\mathbf{b}+\mathbf{b}^{b} .
$$

The remanent field $\mathbf{b}^{r}$ can be expressed in terms of $\dot{\mathbf{h}}^{r}$ through the dissipation potential $\mathscr{D}$ via

$$
\mathbf{b}^{r}=\frac{\partial \mathscr{D}}{\partial \dot{\mathbf{h}}^{r}} .
$$

Equation (11) and (12) lead to the Generalized Standard Material $^{54,55}$ (GSM) relation

$$
\rho_{0} \frac{\partial \psi}{\partial \mathbf{h}^{r}}+\frac{\partial \mathscr{D}}{\partial \dot{\mathbf{h}}^{r}}=0
$$


The above constitutive relations can of course be obtained in an inverse manner, i.e., starting by the GSM framework and work out the various equations resulting from that. Note that $\mathscr{D}$ must be a convex function of $\dot{\mathbf{h}}^{r}$ in order to satisfy the inequality (10) and thus lead to positive magnetic work. In the following, we propose a specific functional form of $\mathscr{D}$.

The energy dissipated during the magnetic domain switching is modeled by use of a convex power-law dissipation potential. This potential serves as the macroscopic phenomenological representation of the energy associated with the collective irreversible domain wall motions ${ }^{56,57}$ at the microscale. In particular, we consider a visco-switching response of the magnet and define a strictly convex power-law dissipation potential by ${ }^{58}$

$$
\mathscr{D}\left(\dot{\mathbf{h}}^{r}\right)=\frac{\dot{h}_{0}^{r} b_{c}\left(\xi_{N}\right)}{m+1}\left(\frac{\left|\dot{\mathbf{h}}^{r}\right|}{\dot{h}_{0}^{r}}\right)^{m+1} .
$$

In this expression, $\dot{h}_{0}^{r}$ is a reference $h$-field rate and $m$ is the rate sensitivity parameter taking values between 1 (linear rate-dependent) and 0 (rate-independent switching surface). The convexity of $\mathscr{D}$ in (14) guarantees a positive magnetic work. In order to model complex loading histories as well as non-trivial hardening responses, the coercive field $b_{c}$ is considered to be a function of $N$ history-dependent variables, denoted compactly by using the set $\xi_{N}$. The elements of $\xi$ will be defined later in the Sections III B and IV A. The scalar parameter, $b_{c}$, serves to describe in a phenomenological manner the coercivity mechanisms found in different permanent magnets and is discussed in detail in Section IIIB.

The Legendre transform of the strictly convex $\mathscr{D}\left(\dot{\mathbf{h}}^{r}\right)$ with respect to $\dot{\mathbf{h}}^{r}$ is defined by

$$
\mathscr{D}^{*}\left(\mathbf{b}^{r}\right)=\sup _{\dot{\mathbf{h}}^{r}}\left[\mathbf{b}^{r} \cdot \dot{\mathbf{h}}^{r}-\mathscr{D}\left(\dot{\mathbf{h}}^{r}\right)\right] .
$$

The maximization operation in the above definition leads to (12), which, in turn, allows to compute $\dot{\mathbf{h}}^{r}$ in terms of $\mathbf{b}^{r}$ and substitute it back to (14) to obtain

$$
\mathscr{D}^{*}\left(\mathbf{b}^{r}\right)=\frac{\dot{h}_{0}^{r} b_{c}\left(\xi_{N}\right)}{n+1}\left(\frac{\left|\mathbf{b}^{r}\right|}{b_{c}\left(\xi_{N}\right)}\right)^{n+1},
$$

or

$$
\dot{\mathbf{h}}^{r}=\frac{\partial \mathscr{D}^{*}}{\partial \mathbf{b}^{r}}=\dot{h}_{0}^{r}\left(\frac{\left|\mathbf{b}^{r}\right|}{b_{c}\left(\xi_{N}\right)}\right)^{n} \mathbf{b}^{r} .
$$

Here, $n=1 / m$ such that $n=1$ leads to a linear ratedependent response. In the limit of $n \rightarrow \infty$, which is of interest in the present work, the dissipation potential becomes convex and reads

$$
\mathscr{D}^{*}\left(\mathbf{b}^{r}\right)= \begin{cases}0, & \left|\mathbf{b}^{r}\right| \leq b_{c}\left(\xi_{N}\right) \\ \infty, & \text { otherwise }\end{cases}
$$

This allows for the definition of the ferromagnetic switching surface ${ }^{14-16}$ which reads

$$
\phi\left(\mathbf{b}^{r}, \xi_{N}\right):=\mathbf{b}^{r} \cdot \mathbf{b}^{r}-b_{c}\left(\xi_{N}\right)^{2} .
$$

This expression corresponds to a spherical switching surface in the $\mathbf{b}^{r}$-space with a radius equal of that prescribed by the coercive field $b_{c}$. The history-dependent variable switching surface (19) is a straightforward extension of existing models in the context of ferroelectric switching $^{14,15}$, where $b_{c}$ is taken to be constant. The evolution of the switching surface with $\xi_{N}$ is discussed in Sections III B and IV B.

Finally, we obtain the associated switching rule from (19) as

$$
\dot{\mathbf{h}}^{r}=\dot{\lambda} \frac{\partial \phi}{\partial \mathbf{b}^{r}}
$$

where $\dot{\lambda}$ is a scalar multiplier with $\dot{\lambda}>0$ when $\phi=0$ and $\dot{\lambda}=0$ when $\phi<0$ (see Appendix for details).

It is relevant to mention here that the present work focuses on the modeling of rate-independent (quasi-static) hysteresis in ferromagnetic materials, i.e. the $n \rightarrow \infty$ limit. Its extension to a rate-dependent response can be obtained by setting $1<n \ll \infty$ in (16). Unfortunately, there exist no experimental data in the literature for ratedependent hysteresis under slowly/moderately changing $h$-field to assess the more general model in those cases.

\section{A. Energetic hardening functions}

The choice of the hardening function is crucial for obtaining an accurate constitutive response. In this section, we propose a specific functional form for $\psi^{r}\left(\mathbf{h}^{r}\right)$ to obtain the back magnetic field $\mathbf{b}^{b}$ in terms of $\mathbf{h}^{r}$. Similar phenomenological models for ferroelectric switching consider the back electric displacement field $\mathbf{d}^{b}$ to be an inverse sigmoid function of the local remanent electric field $\mathbf{e}^{r 13,15-17}$. In this regard, we propose a $\psi^{r}\left(\mathbf{h}^{r}\right)$, which is a combination of a logarithmic and inverse hyperbolic tangent functions such that

$$
\begin{aligned}
\rho_{0} \psi^{r}\left(\mathbf{h}^{r}\right)= & \frac{1}{2} \mathbf{h}^{r} \cdot \boldsymbol{\mu} \cdot \mathbf{h}^{r}-\kappa \psi_{\log }\left(\mathbf{h}^{r}\right) \\
& -(1-\kappa) \psi_{\tanh }\left(\mathbf{h}^{r}\right), \quad 0 \leq \kappa \leq 1,
\end{aligned}
$$

with

$$
\psi_{\log }\left(\mathbf{h}^{r}\right)=K_{1} h_{s}^{r}\left[\log \left(1-\frac{\left|\mathbf{h}^{r}\right|}{h_{s}^{r}}\right)+\frac{\left|\mathbf{h}^{r}\right|}{h_{s}^{r}}\right]
$$

and

$$
\begin{aligned}
\psi_{\tanh }\left(\mathbf{h}^{r}\right)=K_{1} h_{s}^{r} & {\left[\frac{\left|\mathbf{h}^{r}\right|}{h_{s}^{r}} \tanh ^{-1}\left(\frac{\left|\mathbf{h}^{r}\right|}{h_{s}^{r}}\right)\right.} \\
+ & \left.\frac{1}{2} \log \left(\frac{\mathbf{h}^{r}}{h_{s}^{r}} \cdot \frac{\mathbf{h}^{r}}{h_{s}^{r}}-1\right)\right] .
\end{aligned}
$$




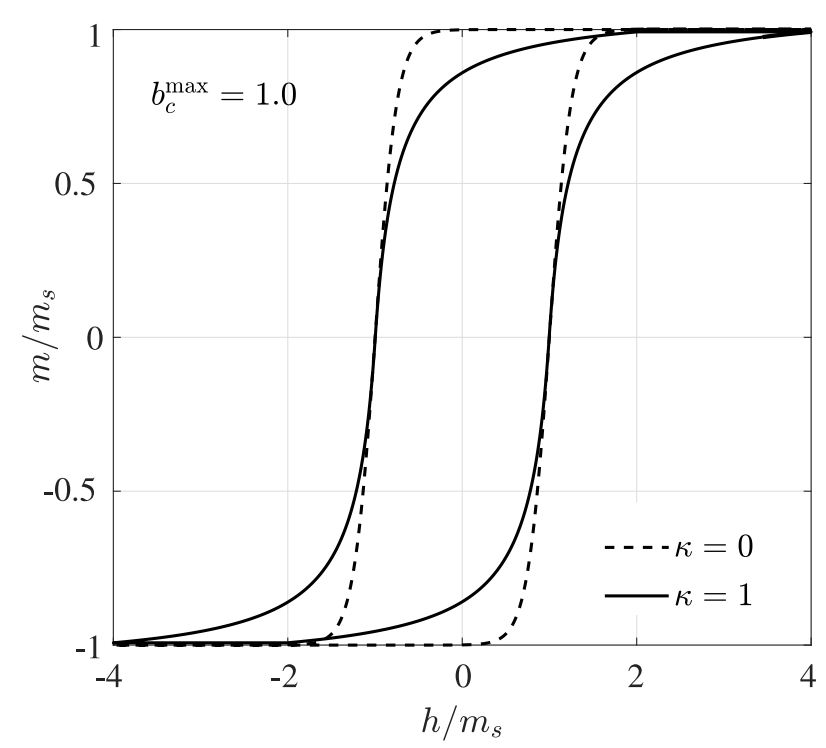

FIG. 1: Hard magnetic response for different $\kappa=0$ and $\kappa=1$.

Here, $h_{s}^{r}$ is the saturation $h^{r}$-field and $K_{1}, \kappa$ are hysteresis shape parameters. In the two special cases corresponding to $\kappa=1$ and $\kappa=0,(21)$ reduces to the hardening functions of Landis ${ }^{14}$ and Klinkel ${ }^{15}$, respectively. Substituting (21), (22) and (23) into (11), we obtain

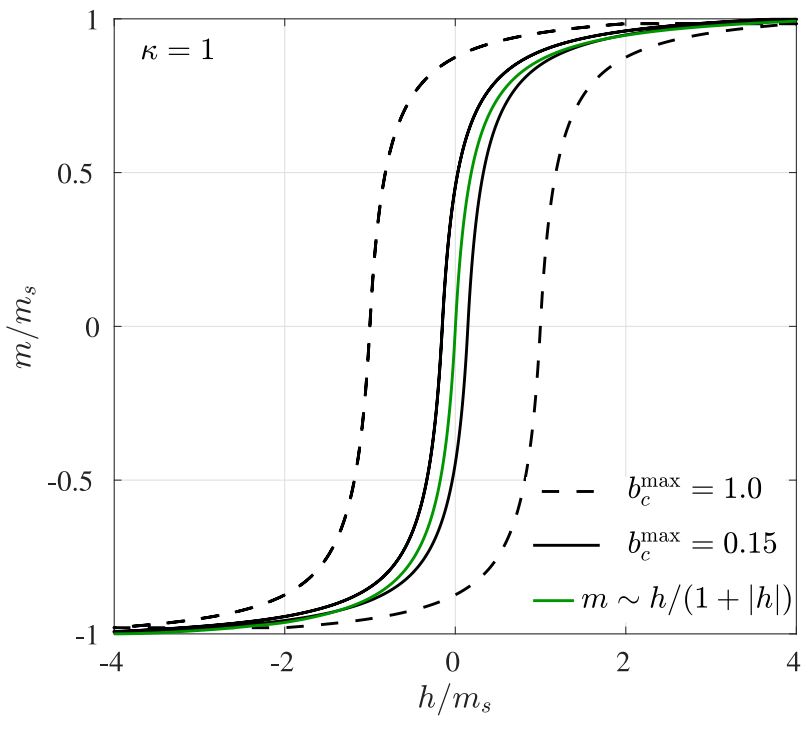

FIG. 2: Soft magnetic response in the limit of $b_{c}^{\max } \rightarrow 0$ and comparison with the corresponding anhysteretic constitutive laws.

Those purely energetic expressions lead to a typical saturating magnetization response in the $m$ - $h$ space. Using (25) along with (2), we obtain the magnetization

$$
\begin{aligned}
& \mathbf{b}^{r}=-\boldsymbol{\mu} \cdot \mathbf{h}-K_{1}\left[\kappa \frac{\mathbf{h}^{r} / h_{s}^{r}}{1-\left|\mathbf{h}^{r}\right| / h_{s}^{r}}+(1-\kappa) \tanh ^{-1}\left(\frac{\left|\mathbf{h}^{r}\right|}{h_{s}^{r}}\right) \frac{\mathbf{h}^{r}}{\left|\mathbf{h}^{r}\right|}\right] . \\
& (24)
\end{aligned} \quad \mathbf{m}=\left\{\begin{array}{l}
m_{s} \tanh \left(\frac{\mu}{K_{1}}|\mathbf{h}|\right) \frac{\mathbf{h}}{|\mathbf{h}|}, \quad \kappa=0 \\
\left.\begin{array}{l}
\text { Depending on whether } \kappa=1 \text { or } 0,(24) \text { reduces to } \\
\text { an inverse sigmoid function of the type } x /(1-|x|) \text { or }
\end{array} \quad \begin{array}{l}
\mu \mathbf{h} / K_{1} \\
1+\mu|\mathbf{h}| / K_{1}
\end{array}\right), \quad \kappa=1,
\end{array}\right.
$$
$\tanh ^{-1}(x)$, respectively. The last relation along with (19) and (20) are used to compute the evolution of $\mathbf{h}^{r}$ for a given variation of the applied magnetic field $\mathbf{h}$ (see Appendix for details).

For illustration purposes, we show in Fig. 1 entire $m-h$ hysteresis loop as obtained by the previously discussed definitions. We apply $\mathbf{h}=h \widehat{\mathbf{e}}_{1}$ (such that $\left.\mathbf{h}^{r}=h^{r} \widehat{\mathbf{e}}_{1}\right)$ and consider the two special cases $\kappa=1$ and $\kappa=0$ corresponding to (22) and (23), respectively. We find a sharper increase of $m$ when $\kappa=0$, whereby $\kappa=1$ leads to a smoother S-shaped hysteresis loop. In practice, the choice of the interpolation parameter $0 \leq \kappa \leq 1$ is made in accord with the available experimental $m$ $h$ response of the magnet, as discussed in detail in the Results Section V.

Before we proceed further, it is useful to introduce the anhysteretic approximations of the above proposed dissipative response. For simplicity, we assume an isotropic magnetic response with $\boldsymbol{\mu}=\mu \mathbf{I}$ and write ${ }^{51}$

$$
\mathbf{b}= \begin{cases}\mu\left[\mathbf{h}+h_{s}^{r} \tanh \left(\frac{\mu}{K_{1}}|\mathbf{h}|\right) \frac{\mathbf{h}}{|\mathbf{h}|}\right], & \kappa=0 \\ \mu\left[\mathbf{h}+h_{s}^{r}\left(\frac{\mu \mathbf{h} / K_{1}}{1+\mu|\mathbf{h}| / K_{1}}\right)\right], & \kappa=1\end{cases}
$$

where

$$
m_{s}=h_{s}^{r} /\left(1-\chi^{(r)}\right)
$$

is the saturation magnetization. The parameter $\chi^{(r)}=$ $1-\mu_{0} / \mu$ denotes the relative susceptibility and $\mu_{0}=$ $4 \pi \times 10^{-7}$ is the permeability of air. Such an anhystrertic constitutive response is typically employed in the modeling of soft magnets like the carbonyl iron powder ${ }^{51}$ or cobalt ferrite ${ }^{59}$. Note that, the purely energetic approximation of (25) for weakly dissipative magnets can be obtained by considering $b_{c} \rightarrow 0$ in (19). However, such an approximation is only a mathematical operation that allows to recover equation (25). This has no physical ground since in practice there exist no magnet, whose $b_{c}$ is identically zero. As shown in Fig. 2, the soft (anhysteretic) magnetic response can be readily obtained from the proposed dissipative framework by use of small values of $b_{c}^{\max }$. Those weakly hysteretic curves can be approximated by the corresponding anhysteretic constitutive laws (26) for simplicity in analysis. Figure 2 clearly indicates that as $b_{c}^{\max }$ goes to zero, the constitutive law reduces to that in equation (26). 


\section{B. Isotropic hardening and symmetric cyclic loading}

In this section, we discuss in detail the evolution of the coercive field $b_{c}\left(\xi_{N}\right)$ in terms of two history-dependent internal variables $(N=2)$, namely, $\xi_{2} \equiv\left\{\bar{h}^{r}, R_{\mathbf{h}^{r}}\right\}$. The first element of $\xi_{2}$ is the accumulated remanent $h$-field (similar to the accumulated plastic strain in mechanical plasticity), defined as

$$
\dot{\bar{h}}^{r}=\sqrt{\dot{\mathbf{h}}^{r} \cdot \dot{\mathbf{h}}^{r}}, \quad \bar{h}^{r}=\int_{t} \dot{\bar{h}}^{r} \mathrm{~d} t .
$$

The second element of $\xi_{2}$ is the radius of the memory surface, which is discussed later in this section.

\section{Initial magnetization and hardening}

It is important to note at this point that in spite of exhibiting a qualitatively similar major hysteresis loop, the underlying mechanisms behind the ferroelectric and ferromagnetic switching differ significantly. The ferroelectrics undergo a phase transition upon loading beyond a critical electric field leading to switching of the polarization direction within a ferroelectric crystal ${ }^{37}$.

On the other hand, the ferromagnets consist of a large number of magnetic domains with different directions of magnetization, whereas domain nucleation and domain wall pinning are the two key mechanisms that cause coercivity. Experiments and micromagnetic theories suggest that the coercivity in RE magnets arise due to a combined effect of domain wall pinning and nucleation ${ }^{60}$. It is observed through advanced imaging techniques that reversed domains nucleate right from the beginning of magnetization of a virgin NdFeB specimen ${ }^{24}$. Thus, a combination of nucleation and pinning causes considerable amount of initial magnetization as compared to a solely pinning-type magnet. In order to capture this microscopic phenomena at the macroscopic level, we consider that the switching surface (19) evolves from a very small radius $b_{c}^{0}$ to a constant limiting surface of radius $b_{c}^{\max }$, which is the saturation coercive field, as shown in Fig. 3a(inset). Motivated by experimental results then, the switching surface radius is a function of $\bar{h}^{r}$ (i.e., the first element of $\xi_{2}$ ) and can take the following form

$$
b_{c}\left(\bar{h}^{r}\right)=b_{c}^{\max }\left\{\tanh \left[\left(\begin{array}{c}
\bar{h}^{r} \\
\bar{h}_{0}^{r}
\end{array}\right)^{8}\right]\right\}^{1 / q} .
$$

In this expression, $\bar{h}_{0}^{r}$ is a reference accumulated remanent field (in analogy to the yield strain in mechanical plasticity) and $q$ is a hardening exponent. The above evolution law for the switching surface is qualitatively similar to the isotropic hardening rules in mechanical plasticity $^{46,47}$. As a consequence of the constitutive relation (29), the magnetic domain nucleation is now captured by allowing switching right from the beginning. Obviously, the hardening exponent $q$ plays a pivotal role in the modeling of different coercivity mechanisms at the macroscopic level. For a better understanding of this parameter, we discuss a few representative cases in the context of Fig. 3. We note first that for $q \rightarrow \infty$, the coercive field $b_{c}\left(\bar{h}^{r}\right) \rightarrow b_{c}^{\max }$, which, indeed, models the pinning type magnets. Thus, in the limit of $q \rightarrow \infty$, the proposed model reduces to the existing ferroelectric switching models ${ }^{13-17}$ with no isotropic hardening.

On the other hand, a pure nucleation-type response is obtained for $q=1$. In this case, as depicted in in Fig. 3a,b, the switching surface starts growing from a radius $b_{c} \approx 0$ at (a) and then evolves through intermediate switching surfaces like (b) to finally saturate to the limiting surface of radius $b_{c}^{\max }$ at (c). Practically, ferromagnets are modeled by choosing values in the range $1 \leq q<\infty$ as there exist no magnet that exhibits only pure domain pinning or only pure domain nucleation.

\section{Cyclic loading and symmetric minor loops}

The symmetric minor loops are obtained whenever the loading/unloading amplitude is kept below the saturation $h$-field $h_{s}$. Note that the magnetic domains do not become fully aligned to the loading direction before saturation. Thus, if a load reversal takes place at $|\mathbf{h}|<h_{s}$, then a lower $h$-field is required to reverse the specimen's magnetization direction. The minor loops during the initial magnetization of a specimen can be obtained in the proposed phenomenological framework by suitably defining the accumulated remanent field $\bar{h}^{r}$. Note further that $\bar{h}^{r}$ is a strictly increasing, history-dependent variable, which controls the evolution of the coercive filed $b_{c}$. In order to capture the symmetric minor loops, we introduce a new history-dependent internal variable $R_{\mathbf{h}^{r}}$ (i.e., the second element of $\xi_{2}$ ) that memorizes the prior maximum remanent field range. The notion of such a remanent field range memory variable was first introduced by Chaboche, Van, and Cordier ${ }^{46}$ in the context of cyclic mechanical plasticity. Following Chaboche ${ }^{47}$, we allow $\dot{\bar{h}}^{r}$ to evolve following equation (29) only if the state of $\mathbf{h}^{r}$ lies on the memory surface. The latter is a spherical surface (for isotropic magnets) of radius $R_{\mathbf{h}^{r}}$ defined in the $\mathbf{h}^{r}$-space by

$$
\phi_{m}:=\mathbf{h}^{r} \cdot \mathbf{h}^{r}-R_{\mathbf{h}^{r}}^{2} .
$$

At a given instant $t_{0}, R_{\mathbf{h}^{r}}$ corresponds to the maximum amplitude that $\mathbf{h}_{r}$ has reached over the entire loading history up to that time instant, i.e.,

$$
R_{\mathbf{h}^{r}}=\max \left\|\mathbf{h}^{r}(t)\right\|, \quad \forall 0<t<t_{0} .
$$

The isotropic hardening is effective only if the local state of $\mathbf{h}^{r}$ lies on the memory surface $\phi_{m}$. This feature is incorporated in the hardening rule (29) by modifying (28) as

$$
\dot{\bar{h}}^{r}= \begin{cases}\sqrt{\dot{\mathbf{h}}^{r} \cdot \dot{\mathbf{h}}^{r}}, & \text { if } \phi_{m}=0 \\ 0, & \text { if } \phi_{m}<0 .\end{cases}
$$



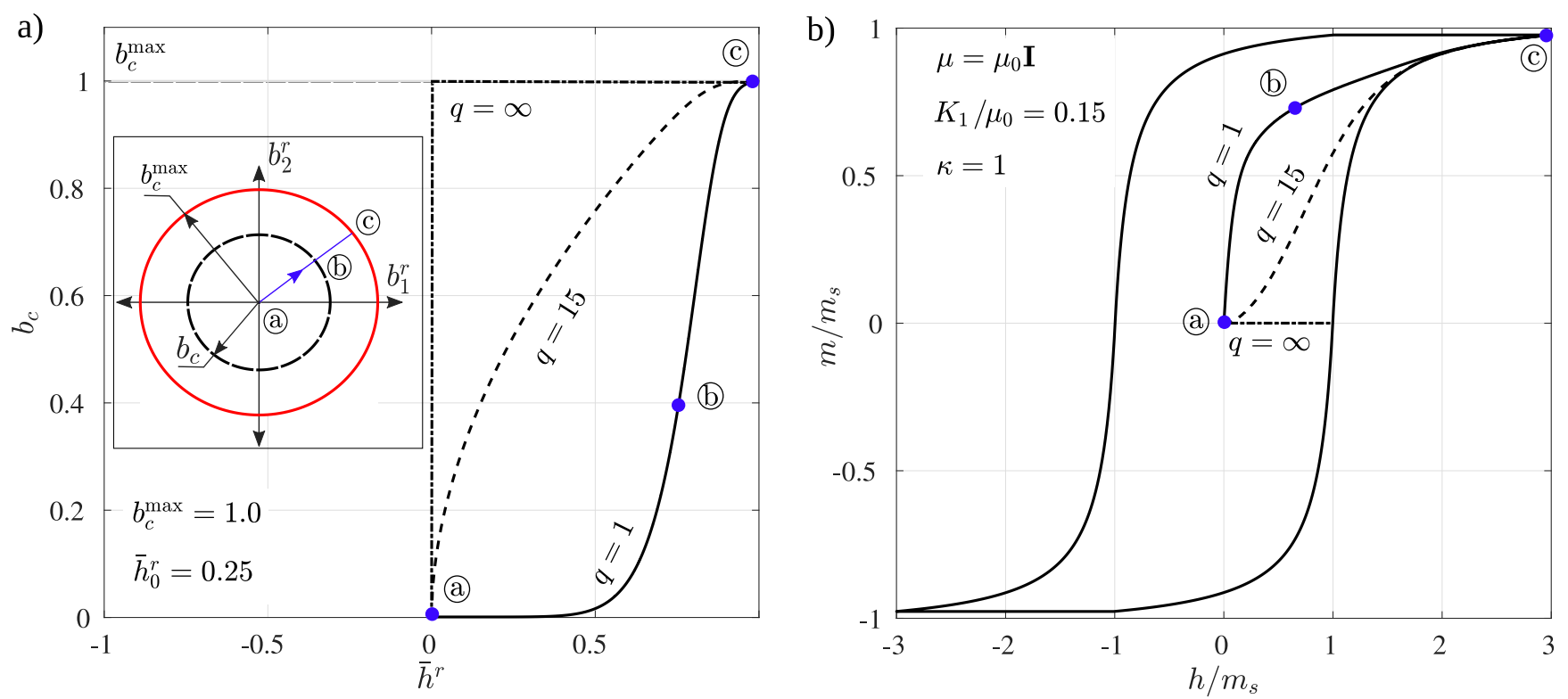

FIG. 3: (a)(inset) Evolving switching surface (in 2D) from a radius of $b_{c}^{0}$ to $b_{c}^{\max }$. (a) Variation of $b_{c}$ with $\bar{h}^{r}$ for different hardening exponents. (b) Effect of hardening exponent $q$ on the initial magnetization curve.

Note that $b_{c}$ in (29) is not an explicit function of $R_{\mathbf{h}^{r}}$. However, $R_{\mathbf{h}^{r}}$ controls the evolution of $\bar{h}^{r}$ through (30) and (32). Thus, there exists an implicit dependence of $b_{c}$ on the second element of $\xi_{2}$, i.e., $R_{\mathbf{h}^{r}}$.

For a better understanding of the modified isotropic hardening law, we discuss a representative example of magnetic cyclic loading with increasing amplitude in Fig. 4. Temporal variation of $h / m_{s}$ is shown in Fig. 4a (inset). Specifically, we observe in Fig. 4a that $\bar{h}^{r}$ increases from (a) to (b). This increase in $\bar{h}^{r}$ results in the evolution of the switching surface radius $b_{c}$ from (a) to (b) (see Fig. 4b (inset)), which leads to the $m-h$ response (a) - (b) in Fig. 4b. Then, unloading from (b) to (d) (beyond $\left.\left|h^{(\mathfrak{b}}\right|=\left|h^{(}\right|\right)$results in two distinct regimes. Firstly, from (b) to (C), $h / m_{s}$ remains less than the maximum loading amplitude of the previous half cycle (a) - (b), i.e., $|h|<\left|h^{(\mathbb{b})}\right|=\left|h^{\complement}\right|$. Thus, $\bar{h}^{r}$ remains constant between (b) - (C), which results in $b_{c}$ to remain the same from (b) to (c) (see Fig. 4b (inset)). In turn, going from (c) to (d), $|h|>\left|h^{(\mathrm{D})}\right|=\left|h^{(\mathrm{C}}\right|$ of the previous half cycle (a) - (b) and hence, $\bar{h}^{r}$ increases resulting to further increase of the switching surface radius in this regime (see Fig. 4b (inset)). The switching surface radius continues to increase in a similar fashion during the subsequent half cycles. e.g., (d) - (f) until eventually reaching $b_{c}^{\max }$.

\section{THE EXTENDED CONSTITUTIVE MODEL FOR ASYMMETRIC CYCLIC LOADING}

So far in the proposed model, we incorporated the effect of isotropic hardening during the initial magnetization. However, experiments show existence of minor hysteresis loops under complex cyclic loading scenarios occuring after the initial magnetization. Clearly, from (29) and (32), we note that $b_{c}$ evolves either during the initial magnetization or when $h$-field increases beyond the previously maximum absolute value of $|\mathbf{h}|$ attained at a preceding cycle (see e.g. Fig. 4). Nevertheless, following available experimental observations, the switching surface can shrink backwards if more complex minor loop loadings are considered. In view of this, we attempt to enrich further the evolution law for $b_{c}$ to model more complex cyclic loading cases, especially when loadreversals may take place before the local $|\mathbf{h}|$ reaches the maximum $h$-field values attained in the preceding cycles.

\section{A. Notion of a bounding surface}

The approach followed to model such complex minor loops is closely related to the bounding surface idea in mechanical plasticity. In particular, it has been observed by Dafalias and Popov ${ }^{43}$ (see also Chaboche ${ }^{47}$ ) in the context of mechanical plasticity that kinematic and isotropic hardening is not enough to model cyclic plasticity under complex loading conditions. In that regard, Dafalias and Popov ${ }^{44}$ proposed a model where the evolution of the plastic internal variables (the remanent internal variable $\mathbf{h}^{r}$ in our case) depends additionally on some discrete, history-dependent parameters associated with loading/unloading of the material. These history parameters are obtained in the present work by constructing a bounding surface in the $\mathbf{h}^{r}$ space. In the following, we first develop the notion of the bounds in $\mathbf{h}^{r}$ for uniaxial loading/unloading and define the historydependent parameters. Subsequently, the notion is ex- 

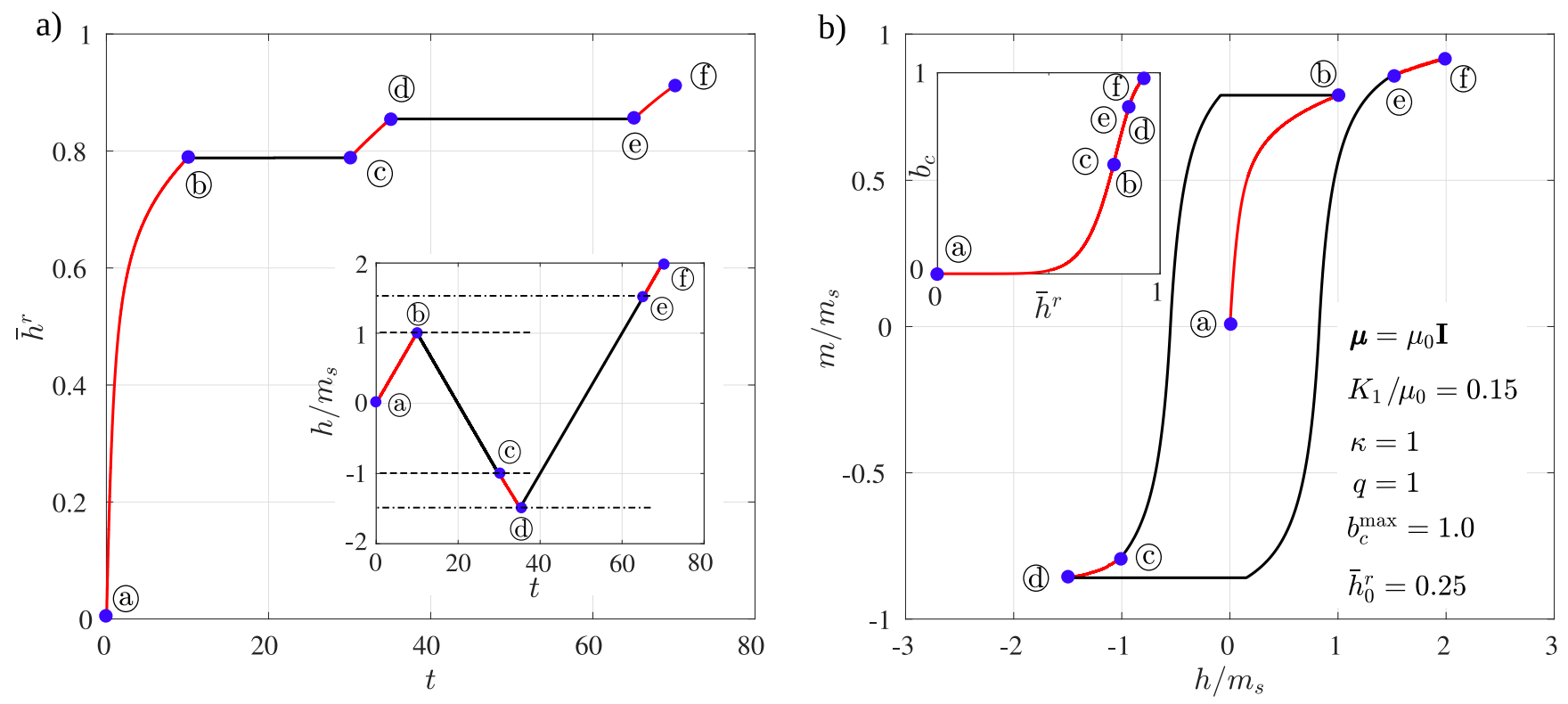

FIG. 4: (a) (inset) Cyclic loading profile. (a) Variation of $\bar{h}^{r}$ with time. (b) (inset) Evolution of $b_{c}$ with $\bar{h}^{r}$. (b) Resulting hysteresis loop.

tended in the context of general three-dimensional multiaxial loading/unloading cases.

A typical schematic representation of a $h^{r}-h$ loop, observed in a uniaxial (1D) experiment is shown in Fig. 5. In this figure, the $h^{r}-h$ loop is observed to remain confined between two bounds: $\left|h^{r}\right| \leq h_{s}^{r}$, namely, an upper and a lower bound. It is observed that from an initial energetic regime $\left(\left|\dot{h}^{r}\right|=0\right)$, the remanent $h$-field switches and eventually saturates to the corresponding bound $\left(\left|h^{r}\right|=h_{s}^{r}\right)$, i.e, the lower bound for the loading half-cycle and the upper bound for the unloading halfcycle, as shown in Fig. 5 for an 1D loading example. Studying numerous uniaxial experiments and following Dafalias and Popov ${ }^{44}$ and Chaboche ${ }^{47}$ we conclude that the minor hysteresis loops can be modeled by considering $b_{c}$ to be a function of the proximity of the current state of remanent field $h^{r}$ to the corresponding bounding (upper/lower) limit. This distance $\delta=A A^{\prime}$, as shown in Fig. 5 is computed by

$$
\delta=h_{s}^{r}+\operatorname{sign}(\dot{h}) h^{r} .
$$

The proximity of a state of $h^{r}$ to the corresponding bounding limit at the beginning of the $p^{\text {th }}$ half-cycle is denoted by $\delta_{(p)}$. The illustrative example of Fig.5 shows $\delta_{(p)}$ for three half-cycles, namely $p=1,2$ and 3 , which together constitute a partially-reversed loading cycle. These initial proximity parameters, $\delta_{(p)}$, are the historydependent terms, which play a pivotal role in modeling the evolution of $b_{c}$ for complex loadings. It is worth noting that the initial local magnetization at the beginning of a new half-cycle also plays a crucial role in the micromagnetic model of Fulmek and Hauser ${ }^{61}$, which also models the minor loops by evolving a history-dependent material parameter. The same notion of evolving the

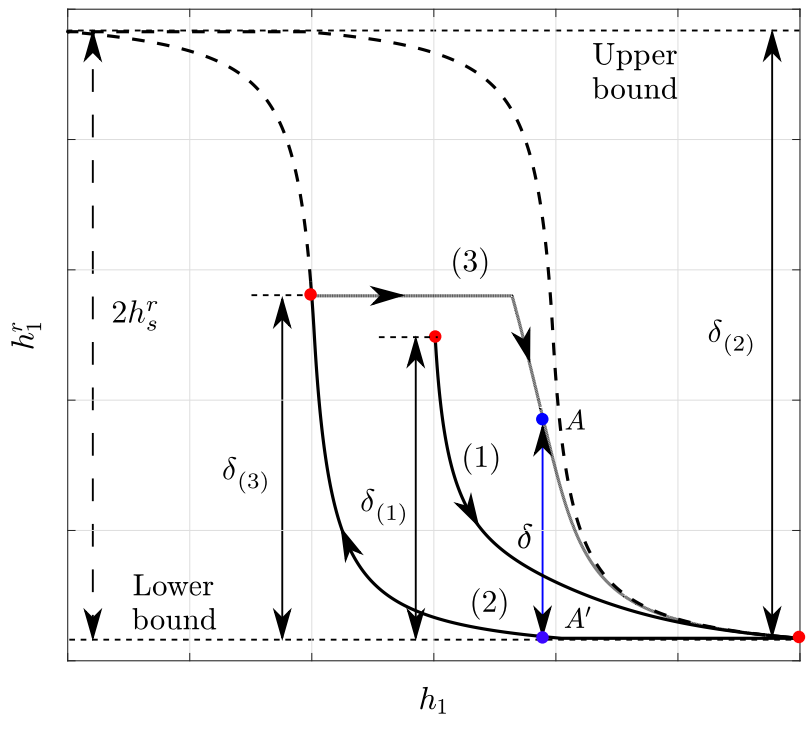

FIG. 5: Schematic illustration of upper and lower bounds, $\delta$ and $\delta_{(p)}$ for the uni-axial loading case.

history-dependent coercive field $b_{c}$ is considered in our model, but within the framework of the bounding surface idea, which is by default a three-dimensional framework and in principle can also be extended to anisotropic magnetic responses.

In this regard, the computation of $\delta$ for the multiaxial case is a bit more involved but otherwise a direct extension of the above described 1D case. For simplicity, we assume an isotropic magnetic response to obtain a spherical bounding surface $\mathcal{B}$ in the $\mathbf{h}^{r}$ space of diameter $2 h_{s}^{r}$ (see Fig. 6a). To obtain $\delta$, we first construct a plane 

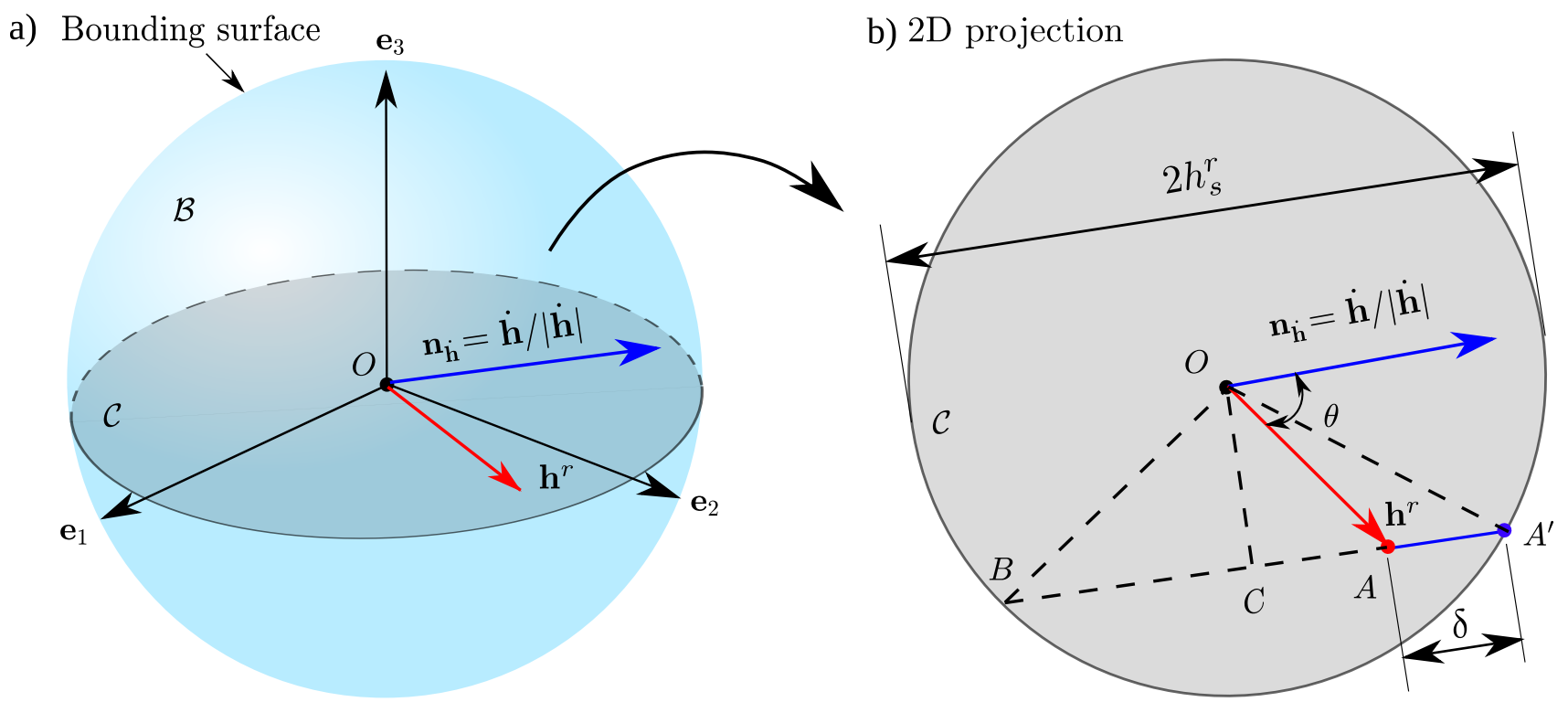

FIG. 6: (a) Schematic illustration of the bounding surface $\mathcal{B}$ and the circle $\mathcal{C}$ on the plane containing the vectors $\mathbf{n}_{\mathbf{h}}$ and $\mathbf{h}^{r}$. (b) Estimation of $\delta_{(p)}$ from the planar geometry on $\mathcal{C}$.

containing both the vectors $\mathbf{h}^{r}$ and $\mathbf{n}_{\dot{\mathbf{h}}}=\dot{\mathbf{h}} /|\dot{\mathbf{h}}|$ and compute $\theta=\cos ^{-1}\left(\mathbf{h}^{r} \cdot \mathbf{n}_{\dot{\mathbf{h}}} /\left|\mathbf{h}^{r}\right|\right)$. The intersection of this plane with $\mathcal{B}$ leads to a circle $\mathcal{C}$, whose $3 \mathrm{D}$ view and the 2D projection are shown in Fig. 6a and b, respectively. Now, $\delta$ is obtained by computing the Eucledian distance between $A A^{\prime}$, where $A$ represents the current state of $\mathbf{h}^{r}$ and $A^{\prime}$ is the corresponding point on $\mathcal{B}$ obtained by extending a straight line from $A$ in the direction of $\mathbf{n}_{\mathbf{h}}$ (see Fig. 6b). From the planar geometry on the circle $\mathcal{C}$ (Fig. 6b) with center $O$ and radius $h_{s}^{r}$, we obtain $\delta$ using straightforward geometrical arguments as

$$
\delta=\sqrt{\left(h_{s}^{r}\right)^{2}-\left|\mathbf{h}^{r}\right|^{2} \sin ^{2} \theta}+\left|\mathbf{h}^{r}\right| \cos \theta .
$$

Figure $6 \mathrm{~b}$ shows that the uniaxial loading and unloading cases presented in Fig.5 and defined via the equation (33) are special cases of the more general three-dimensional framework that can be obtained by setting $\theta=0$ and $\pi$, respectively, in equation (34).

By summarizing the above discussion, one may define the history-dependent variables to form a set of size $2 p+$ 4 , i.e.,

$\xi_{2 p+4} \equiv\left\{\bar{h}^{r}, R_{\mathbf{h}^{r}}, \bar{h}^{e}, \delta, \delta_{(p)}, \bar{h}_{(p)}^{e}, \delta_{(p-1)}, \bar{h}_{(p-1)}^{e}, \ldots, \delta_{(1)}, \bar{h}_{(1)}^{e}\right\}$,

where $p$ is the number of half cycles (including the current one), and $\bar{h}^{e}$ is the accumulated energetic $h$-field (defined later in (38), similar to $\left.\bar{h}^{r}\right)$. Finally, $\bar{h}_{(p)}^{e}$ is the $\bar{h}^{e}$ at the beginning of the $p^{\text {th }}$ half-cycle (similar to $\left.\delta_{(p)}\right)$. In principle, $\xi$ is a set that has a continuously increasing number of elements, starting from $\xi_{6} \equiv\left\{\bar{h}^{r}, R_{\mathbf{h}^{r}}, \bar{h}^{e}, \delta, \delta_{(1)}, \bar{h}_{(1)}^{e}\right\}$. Fortunately, real materials exhibit a fading memory ${ }^{43,44}$. As a result, one can consider gradually increasing relative weights on $\delta_{(p)}, \delta_{(p-1)}, \delta_{(p-2)}$ and so on and eventually discard the oldest history variables. A specific functional form of $b_{c}\left(\xi_{2 p+4}\right)$ is proposed next based on available experimental observations.

It should be noted that in the general context of magnetically anisotropic materials the bounding surface $\mathcal{B}$ is expected to become non-spherical albeit remain convex. The proposed framework can be readily extended for such cases but would probably require additional variables and anisotropic invariants.

\section{B. First order minor loops}

Typical experiments ${ }^{39,40,62}$ show that the first order minor loops (FOMLs) exhibit, in a sense, two distinct features; (i) a low $|\mathbf{h}|$ switching depends on the loading/unloading history while (ii) the switching surface radius increases from $b_{c}$ to $b_{c}^{\max }$ during subsequent switching. When a load reversal takes place at a $|\mathbf{h}|<h_{s}$, both the rotation of the magnetic domains in the previous loading direction and the pinning of domain walls remain incomplete. Thus, one observes switching at a lower $|\mathbf{h}|$ during the current half cycle because of partial rotation of the magnetic domain walls during the previous half cycle $^{57}$. Then, as switching proceeds during the current half cycle, the domain walls get rotated and pinned in the direction of $|\mathbf{h}|$, as usual. This micromagnetic phenomenon is captured in the present phenomenological model as follows; $b_{c}$ is allowed to decrease during the energetic response of a partially-reversed half-cycle, whereby it may increase up to $b_{c}^{\max }$ during a subsequent switching. By recalling the observations (i) and (ii) done previously, we combine all the previous constitutive equations for $b_{c}$ and extend them into three distinct evolution laws for the switching surface for the three different 
regimes - (i) the initial magnetization, (ii) the energetic part, and (iii) the switching part of a half cycle. Each one of these regimes is considered independently in the present work and their modeling can be carried separately depending on the level of complexity one is willing to reach.

Specifically, the initial magnetization regime is indicated by a function

$$
\mathcal{K}=\mathcal{H}\left(\dot{\bar{h}}^{r}\right)
$$

which is essentially a Heaviside step function with $\mathcal{K}=1$ during the initial magnetization and 0 otherwise. Simi- larly, the energetic and the switching regimes of a halfcycle are written in terms of a Heaviside step function as

$$
\mathcal{J}=1-\mathcal{H}\left(1-\frac{\delta}{\delta_{(p)}}\right)
$$

Recall from Section IV A that $\delta=\delta_{(p)}$ during the energetic response and $\delta$ starts decreasing from $\delta_{(p)}$ when the switching starts. Thus, from definition (36), we have $\mathcal{J}=1$ and 0 to represent the energetic and the switching regimes, respectively. We now propose a combined evolution law for $b_{c}$ depending on the indicator functions $\mathcal{K}$ and $\mathcal{J}$ such that

$$
b_{c}= \begin{cases}b_{c}^{\max }\left\{\tanh \left[\left(\frac{\bar{h}^{r}}{\bar{h}_{0}^{r}}\right)^{8}\right]\right\}^{1 / q} & \text { if } \mathcal{K}=1 \\ b_{c}^{(0)}\left\{1-k_{1}\left(1-\frac{\delta_{(p)}}{2 h_{s}^{r}}\right) \frac{\left.R_{\mathbf{h}^{r}} \mathscr{F}_{1}\right\}}{h_{s}^{r}}\right. & \text { if } \mathcal{K}=0, \mathcal{J}=1 \\ b_{c}^{(0)}+\left[b_{c}^{\max }\left\{\tanh \left[\left(\frac{\bar{h}^{r}}{\bar{h}_{0}^{r}}\right)^{8}\right]\right\}^{1 / q}-b_{c}^{(0)}\right] \mathscr{F}_{2} & \text { if } \quad \mathcal{K}=0, \mathcal{J}=0,\end{cases}
$$

where $k_{1}$ is a material parameter, $b_{c}^{(0)}$ is the initial $b_{c}$ at the beginning of the energetic/switching regime, while the first law corresponding to the initial magnetization is the one proposed in (29).

The functions $\mathscr{F}_{1}$ and $\mathscr{F}_{2}$ are used to ensure a smooth decrease/increase in $b_{c}$ during the energetic and switching parts, respectively, of a partially-reversed half-cycle. Note that during the energetic response, i.e., when the switching surface is modeled to shrink following $(37)_{2}$, both the accumulated remanent field $\bar{h}^{r}$ and the proximity parameter $\delta$ remain constant. Thus, the evolution of $\mathscr{F}_{1}$ during the energetic response may be carried out in terms of an accumulated energetic $h$-field, defined as

$$
\dot{\bar{h}}^{e}=\sqrt{\dot{\mathbf{h}}^{e} \cdot \dot{\mathbf{h}}^{e}}, \quad \bar{h}^{e}=\int_{t} \dot{\bar{h}}^{e} d t .
$$

A straightforward choice is to consider $\mathcal{F}_{1}$ to be a saturation function, which evolves from 0 and saturate at 1 , such as

$$
\mathscr{F}_{1}=\tanh \left\{\frac{\delta_{(p)}^{D}}{2 R_{\mathbf{h}^{r}}}\left(\frac{\bar{h}^{e}}{\bar{h}_{0}^{e}}-\frac{\bar{h}_{(p)}^{e}}{\bar{h}_{0}^{e}}\right)\right\}
$$

where the term $\bar{h}_{(p)}^{e}$ is a history-dependent parameter, that is $\bar{h}^{e}$ at the beginning of the $p^{\text {th }}$ half cycle (similar to $\left.\delta_{(p)}\right)$ and $\delta_{(p)}^{D}=\delta_{(p)}+\delta_{(p-1)}-2 h_{s}^{r}$ is the proximity of the $p^{\text {th }}$ half-cycle to the $(p-1)^{\text {th }}$ one, i.e., the proximity between two subsequent half-cycles. Finally, $\bar{h}_{0}^{e}$ in (39) is a material parameter that represents a reference accumulated energetic $h$-field (similar to $\bar{h}_{0}^{r}$ in (29)). Similarly,
$\mathscr{F}_{2}$ is chosen to be a saturation function, that evolves from 0 to 1 , such that

$$
\mathscr{F}_{2}=\tanh \left\{k_{2} \frac{2 h_{s}^{r}}{\delta^{M}}\left(1-\frac{\delta}{\delta_{(p)}}\right)\left(\frac{\delta_{(p)}^{D}}{2 h_{s}^{r}}\right)^{-k_{3}}\right\},
$$

where $k_{2}$ and $k_{3}$ are positive parameters that dictate the shape of the minor loop, and

$$
\delta^{M}=\max \left\{\delta_{(1)}, \delta_{(2)}, \ldots, \delta_{(p)}\right\} .
$$

Note that $\mathscr{F}_{2}$ may become singular in the degenerate case of $\delta_{(p)}^{D} \rightarrow 0$, i,e. for tiny minor loops due to small fluctuations in the local $\mathbf{h}$. This can remedied by adding a correction term in $(37)_{2}$. Such a correction is shown in Appendix VIII but is not necessary for well-defined cyclic loads. The use of (37) to modeling the FOMLs - arising due to partially-reversed loading cycles is discussed next by use of two typical examples.

A typical example of a first-order reversal curve is depicted in Fig. 7. The inset of Fig. 7a shows the uniaxial loading profile, where (a) - (b) represents the initial magnetization of a virgin sample followed by a partiallyreversed cycle (b) - (d) - (f). The initial magnetization from (a) to (b) results in the corresponding evolution of $b_{c}$ as evaluated from $(37)_{1}$ (see Fig. 7a). The corresponding response in the $m-h$ space is shownfor the same regime in Fig. $7 \mathrm{~b}$.

At the beginning of the second half-cycle (b) - (d), we have $\delta_{(2)} \approx 2 h_{s}^{r}$ and $b_{c}^{(0)}=b_{c}^{\max }\left\{\tanh \left[\left(\bar{h}^{r} / \bar{h}_{0}^{r}\right)^{8}\right]\right\}^{1 / q}$. Thus, the evolution law for $b_{c}$, i.e, $(37)_{2}$ reduces to 
a)

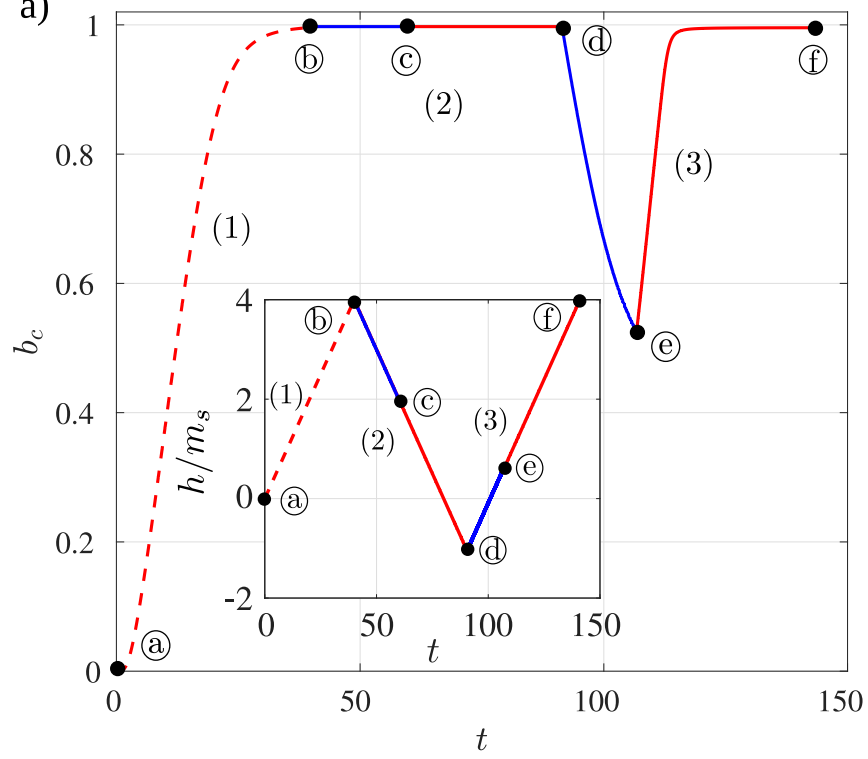

b)

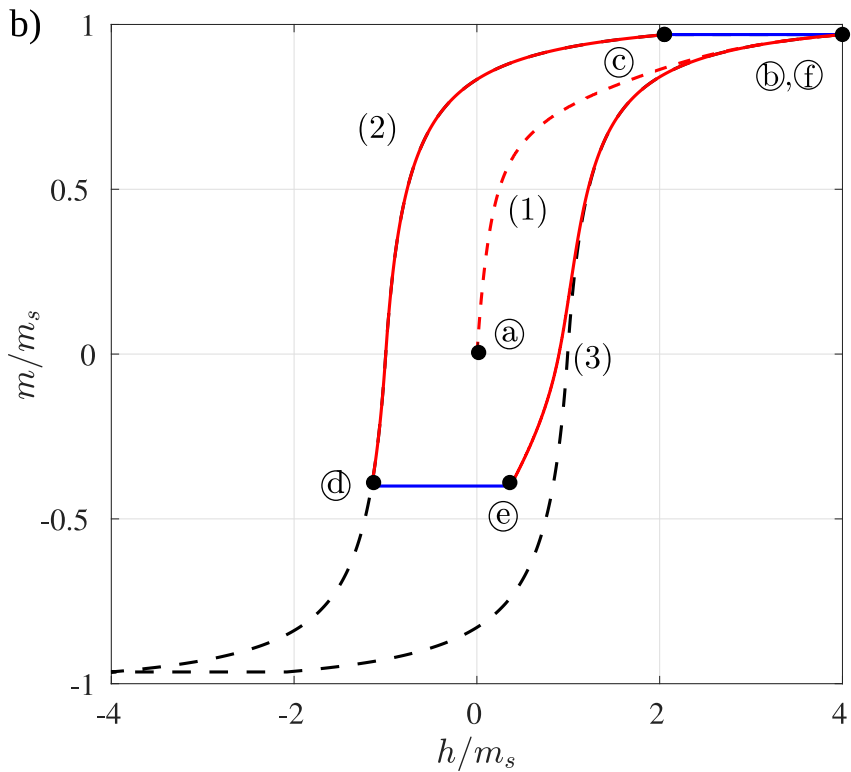

FIG. 7: (a) (inset) Cyclic loading profile. (a) Evolution of $b_{c}$ in time. (b) Resulting $m-h$ loop.

$b_{c}=b_{c}^{(0)}$, resulting in a constant $b_{c}$ between (b) - (C). During subsequent switching, $b_{c}$ evolves following $(37)_{3}$. Note in $(37)_{3}$ that the coefficient of $\mathscr{F}_{2}$ now vanishes as $b_{c}$ did not decrease from $b_{c}^{\max }$. Hence, $(37)_{2}$ and $(37)_{3}$ together ensure a constant $b_{c}$ on unloading if the preceding loading goes beyond saturation (see Fig. 7a). Consequently in Fig. 7b, we obtain a $m-h$ response in the interval (b) - (C) coinciding with the major loop.

The path (d) - (f) represents a partially-reversed halfcycle, where the load reversal takes place before saturation at (d) and thus, $\delta_{(3)}<2 h_{s}^{r}$. During the energetic response, (d) - (e), $b_{c}$ decreases to values lower than $b_{c}^{(0)}=b_{c}^{\max }$ following the constitutive expression $(37)_{2}$ (see Fig. 7a). Consequently, the switching is initiated at (e) at values lower than $b_{c}^{\max }$. From that point on, $b_{c}$ starts evolving following $(37)_{3}$ and hence increases from (e) to (f) approaching $b_{c}^{\max }$. The resulting $m-h$ response is shown by the segment (d) - (e) in Fig. 7b.

Note that the shape of the minor loop (d) - (e) depends explicitly on the choice of the minor loop shape parameters $k_{1}, k_{2}$ and $k_{3}$. The identification of $k_{1}, k_{2}$ and $k_{3}$ by use of a least-square fitting of the model with available experimental data is discussed in Section V. On the other hand, the proposed forms of the functions $\mathscr{F}_{1}$ and $\mathscr{F}_{2}$ will be shown to be sufficient to model a large number of different magnets.

For completeness, we also investigate a more complicated loading scenario where initially the magnet is not magnetized to saturation. That is a very important case in particle-filled magnetorheological elastomers, whereby not all particles attain the same level of magnetization given an external overall magnetic applied field.

In particular, the loading profile is depicted in the inset of Fig. 8a. In this case, an initial loading from (a) to (b) increases $b_{c}$ up to a given value $b_{c(1)}$ resulting to an ini- tial magnetization response in the same shown in Fig. 8b. The subsequent two half cycles (b) - (d) and (d) - (f) follow the evolution equations $(37)_{2}$ and $(37)_{3}$ during the corresponding energetic and switching regimes, respectively, and result in a $m-h$ response, shown in Fig. 8b. An interesting observation can be made during the last unloading half-cycle (f) - (1). We notice from Fig. 8a (inset) that $|h|$ exceeds $h^{\max }$ when the magnet is unloaded beyond (h). Thus, the shrinkage $(\mathbb{f}-$ (g) of the switching surface is followed by its expansion in two steps. First, we get the expansion from (g) to (h) evaluated by equation $(37)_{3}$. This is followed by an expansion of $b_{c}$ in the interval (h) to (1) as computed by equation $(37)_{1}$. As a result, we observe a two step magnetization response (g) - (1) in Fig. 8b. Therefore, three different regimes are obtained from relation (37) during a half-cycle for a loading condition in the interval (1) - (1).

Accurate prediction of the higher-order minor loops necessitates a more tedious definition of $b_{c}$ and, in general, it requires a metric to identify the order of the minor loop. On the other hand, the nature of evolution of $b_{c}$ remains the same for the higher order loops except for the need of additional history-dependent terms. Thus, an efficient storage of the order of the minor loop and the set $\xi$ requires a suitable computational algorithm, which is beyond the scope of this paper. However, typically in engineering applications with magnets ${ }^{63}$ and MREs ${ }^{64}$, the material is not loaded with a highly fluctuating magnetic field that may cause secondary or higher order minor loops. Thus, the proposed model that captures accurately the FOMLs, is expected to be sufficient for the applications at hand. 

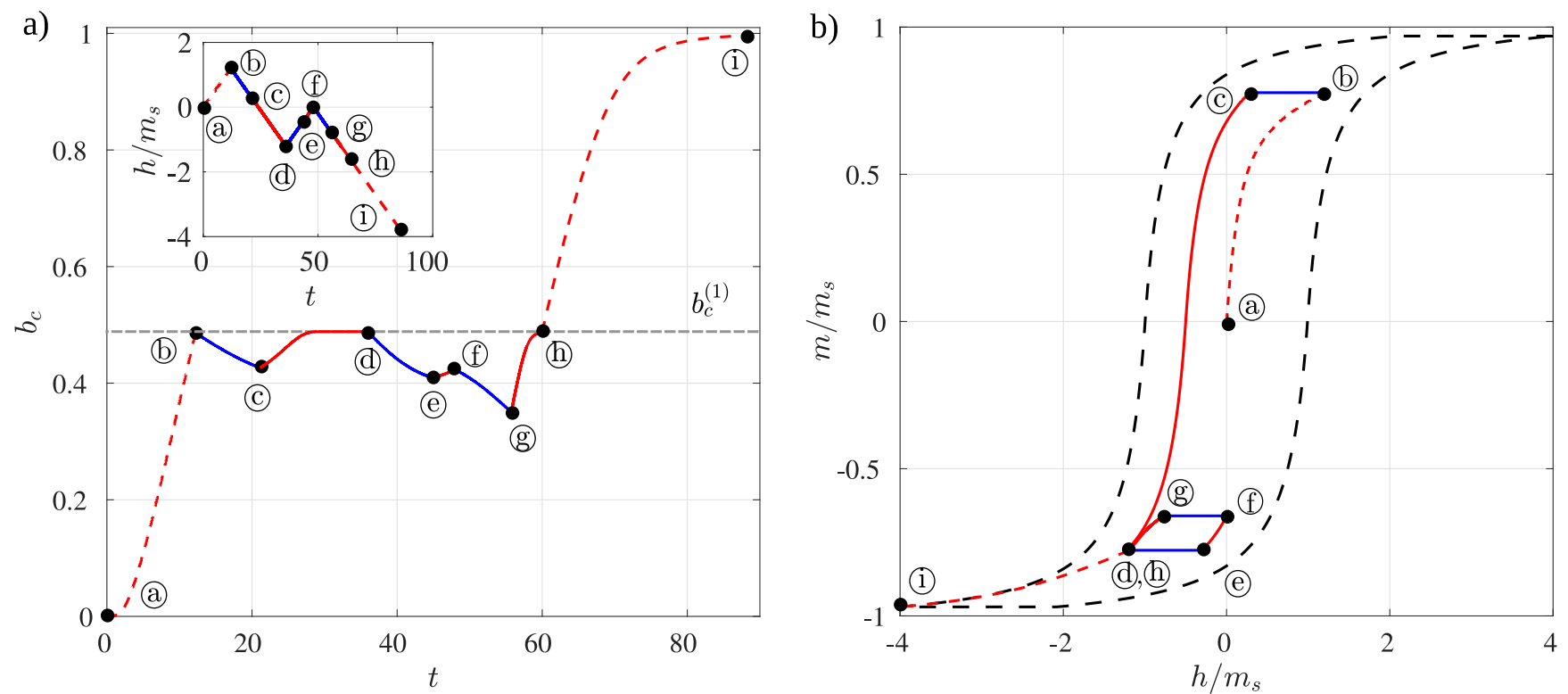

FIG. 8: (a) (inset) Cyclic loading profile. (a) Evolution of $b_{c}$ in time. (b) Resulting $m-h$ loop.

\section{ASSESSMENT OF THE MODEL WITH EXPERIMENTS}

In this section, we use the proposed model to probe and predict a large number of experimental data available in the literature. The number of model parameters to be specified depends on the complexity of the experiment we intend to model. For the most general case which involves modeling a magnet accurately up to its first-order minor loops, we need to specify eleven parameters, namely, $\chi^{(r)}, K_{1}, h_{s}^{r}, \kappa, b_{c}^{\max }, \bar{h}_{0}^{r}, \bar{h}_{0}^{e}, q, k_{1}, k_{2}$ and $k_{3}$. On the other hand, modeling only the major loop requires five material parameters, namely $\chi^{(r)}, K_{1}, h_{s}^{r}$, $\kappa$, and $b_{c}^{\max }$ with all others to remaining inconsequential. Modeling the initial magnetization response along with the major loop requires two additional parameters $\bar{h}_{0}^{r}$ and $q$. Finally, for the modeling of the minor loops, we need to specify the remaining $\bar{h}_{0}^{e}, k_{1}, k_{2}$ and $k_{3}$ constants.

In this regard, we carry out a least square fitting of the model parameter to available experimental data in three steps.

i We first find the optimal major loop parameters $\bar{b}_{c}^{\text {max }}$, $\chi^{(r)}, \bar{K}_{1}$ and $h_{s}^{r}$ by using the lsqcurvefit function in MATLAB ${ }^{65}$. For simplicity, we manually select $\kappa=0$ or 1 , depending on the nature of the hysteresis loop.

ii We, then, find the initial magnetization parameters $q$ and $\bar{h}_{0}^{r}$ using the same function.

iii Finally, we identify the rest of the four minor loop parameters, namely, $\bar{h}_{0}^{e}, k_{1}, k_{2}$ and $k_{3}$ if such information are available from the experiments analyzed.

It is useful at this point to write down the simple form of our model in the case of uniaxial loading (say, along $\mathbf{e}_{1}$ ) of isotropic magnets, which corresponds to most experiments in the literature and those analyzed in the following. Thus, (6) becomes

$$
b=\frac{\mu_{0}}{\left(1-\chi^{(r)}\right)}\left(h-h^{r}\right),
$$

The magnetization is then computed from

$$
\mu_{0} m=b-\mu_{0} h=\mu_{0}\left\{\frac{1}{\left(1-\chi^{(r)}\right)}\left(h-h^{r}\right)-h\right\},
$$

while the uniaxial remanent field is obtained from

$$
b^{r}=\frac{\mu_{0}}{\left(1-\chi^{(r)}\right)}\left\{h+\bar{K}_{1} f\left(\frac{h^{r}}{h_{s}^{r}}\right)\right\} .
$$

Here, $\bar{K}_{1}=K_{1}\left(1-\chi^{(r)}\right) / \mu_{0}$ and $f\left(h^{r}\right)$ are defined in $(24)$. Consequently, the switching surface (19) now becomes

$$
\phi:=\left(b^{r}\right)^{2}-\frac{\mu_{0}^{2}}{\left(1-\chi^{(r)}\right)^{2}} \bar{b}_{c}^{2} .
$$

We compute $h^{r}$ from an associated switching rule, obtained from the above using the classical radial return algorithm by Ortiz and Simo ${ }^{66}$. The algorithm used in our computations is detailed for completeness in the Appendix.

In the following, we compare the proposed model with the uniaxial tests of (a) sintered $\mathrm{NdFeB}$ magnets by Huang et al. ${ }^{6}$ and (b) NdFeB powders by Deng et al. ${ }^{5}$ and Périgo et al. ${ }^{4}$. In those examples, we model the initial magnetization and the major loop. Subsequently, we compare the proposed model with the experimentally observed minor loops by Włodarski ${ }^{39}$ and Liu et al. ${ }^{3}$. It is important to mention that the polycrystalline magnets, used in the corresponding experiments, are typically isotropic. Anisotropic magnets may be manufactured through dedicated manufacturing techniques and 
will require additional constitutive parameters that will allow to describe properly the preferred magnetization directions.

\section{A. Sintered NdFeB magnets}

Sintered $\mathrm{NdFeB}$ magnets are the classical example of nucleation-type magnets with high initial susceptibility. In Fig. 9 we obtain two sets of experimental data from Huang et $a l .{ }^{6}$ - (a) the major hysteresis loop for spark plasma sintered NdFeB magnets, where the solid magnet is obtained by sintering very fine melt-spun NdFeB ribbons of less than $40 \mu \mathrm{m}$ diameter, and (b) the major loop for sintered $\mathrm{NdFeB}$, prepared by sintering coarse NdFeB ribbons (greater than $40 \mu \mathrm{m}$ diameter).

We obtain a least-square fit of our model with the experimental data in Fig. 9a in two steps. First, we fit the outer loop of the experiment and obtain the optimal $\bar{b}_{c}^{\max }, \chi^{(r)}, \bar{K}_{1}$ and $h_{s}^{r}$ as shown in Table 1 . Next, the model is fitted to the experimental initial magnetization response to obtain $\bar{h}_{0}^{r}$ and $q$ as shown in Table I. The minor loop parameters $\bar{h}_{0}^{e} k_{1}, k_{2}$ and $k_{3}$ are inconsequential and hence, are set to $\bar{h}_{0}^{e}=1.0 \mathrm{MA} / \mathrm{m}$ and $k_{1}=k_{2}=k_{3}=0$ due to unavailability of any minor loop data.

TABLE I: Material parameters for sintered NdFeB magnets

\begin{tabular}{ll}
\hline \hline$\chi^{(r)}=0.0748$ & $\bar{K}_{1}=0.28 \mathrm{MA} / \mathrm{m}$ \\
$h_{s}^{r}=0.65 \mathrm{MA} / \mathrm{m}$ & $\kappa=0.0$ \\
$\bar{b}_{c}^{\max }=1.20(a) \mid 1.52(b) \mathrm{MA} / \mathrm{m}$ & $\bar{h}_{0}^{r}=0.58 \mathrm{MA} / \mathrm{m}$ \\
$q=3.35$ & $\bar{h}_{0}^{e}=1.0 \mathrm{MA} / \mathrm{m}$ \\
$k_{1}=0.0$ & $k_{2}=0.0$ \\
$k_{3}=0.0$ & \\
\hline \hline
\end{tabular}

We observe that the present model is capable of probing accurately the experimental data in Fig. 9a. Here, we note that in addition to modeling the major loops, the present model also traces accurately the initial magnetization response. Note that the simple switching surface models of Landis ${ }^{14}$ and Kinkel ${ }^{15}$, shown for comparison as well as the pseudo-particle models only model the outer coercive loops accurately. Next, we probe the hysteresis loop for a coarse-grained sintered NdFeB magnet in Fig. 9b. The model is able to reproduce this second case by only re-identifying the parameter $b_{c}^{\max }$, keeping the rest of the parameters the same. Thus, it is observed that, different variants of magnets made of the same material (e.g., exhibiting different grain sizes) can be modeled by a small variation of the coercive field $b_{c}^{\max }$.

\section{B. NdFeB powder}

In Fig. 10, we consider the experimental data of Deng et al. ${ }^{5}$ and Périgo et al. ${ }^{4}$ for quasi-static, uniaxial load- ing of melt-spun NdFeB powder samples. In both cases, the proposed model is capable of reproducing extremely well the corresponding experimental data (see Table II for corresponding parameters). Specifically, we first identify the model parameters using the experimental results of Deng et al. ${ }^{5}$. Then, the experiments of Périgo et $a l .{ }^{4}$ are probed by re-adjusting $\bar{K}_{3}$ and $\chi^{(r)}$, which serve to describe the shape of the hysteresis loop. Table II displays the values of the model parameters, used to describe the major hysteresis loop. Note that in both experiments the maximum coersive field $b_{c}^{\max }$ is identical.

TABLE II: Material parameters for NdFeB powder

\begin{tabular}{ll}
\hline \hline$\chi^{(r)}=0.095(a) \mid 0.163(b)$ & $\bar{K}_{1}=0.078(a) \mid 0.15(b) \mathrm{MA} / \mathrm{m}$ \\
$h_{s}^{r}=0.67 \mathrm{MA} / \mathrm{m}$ & $\kappa=1.0$ \\
$\bar{b}_{c}^{\max }=0.766 \mathrm{MA} / \mathrm{m}$ & $\bar{h}_{0}^{r}=0.551 \mathrm{MA} / \mathrm{m}$ \\
$q=17.5$ & $\bar{h}_{0}^{e}=1.0 \mathrm{MA} / \mathrm{m}$ \\
$k_{1}=0.0$ & $k_{2}=0.0$ \\
$k_{3}=0.0$ & \\
\hline \hline
\end{tabular}

In addition, use of the same initial magnetization parameters $\bar{h}_{0}^{r}$ and $q$ allows us to recover accurately the initial magnetization response in both samples. In Fig. 10, the parameter $q$ implies that the coercivity in NdFeB powder samples can be attributed to a combined effect of nucleation and pinning, which is in agreement with recent microscopic observations by Liu et al. ${ }^{67}$ in $\mathrm{NdFeB}$ ribbons (from which the NdFeB powders are obtained through grinding). Finally, the four minor loop parameters $\bar{h}_{0}^{e}$, $k_{1}, k_{2}$ and $k_{3}$ are inconsequential due to unavailability of any minor loop data and thus are set to $\bar{h}_{0}^{e}=1.0 \mathrm{MA} / \mathrm{m}$ and $k_{1}=k_{2}=k_{3}=0$.

\section{MgMn steel}

In Fig 11, we use the complete model to probe the experimental data of Włodarski ${ }^{39}$ for MgMn steel, which include several fully-reversed, uniaxial hysteresis loops with different amplitudes of loading. Thus, in addition to the initial magnetization and major coercive loop, the latter experiments provide also information on symmetric minor loops. We first identify $\bar{b}_{c}^{\max }, \chi^{(r)}, \bar{K}_{1}$ and $h_{s}^{r}$ in order to probe the experimental major loop (see Table III).

TABLE III: Material parameters for MgMn Steel

\begin{tabular}{ll}
\hline \hline$\chi^{(r)}=0.9913$ & $\bar{K}_{1}=9.5 \mathrm{~A} / \mathrm{m}$ \\
$h_{s}^{r}=1583.0 \mathrm{~A} / \mathrm{m}$ & $\kappa=0.0$ \\
$\bar{b}_{c}^{\max }=72.0 \mathrm{~A} / \mathrm{m}$ & $\bar{h}_{0}^{r}=1451.0 \mathrm{~A} / \mathrm{m}$ \\
$q=45.6$ & $\bar{h}_{0}^{e}=98.7 \mathrm{~A} / \mathrm{m}$ \\
$k_{1}=3.0$ & $k_{2}=2.5$ \\
$k_{3}=0.1$ & \\
\hline \hline
\end{tabular}

Subsequently, we fit the initial magnetization response by identifying the parameters $q$ and $\bar{h}_{0}^{r}$. Finally, we fit 

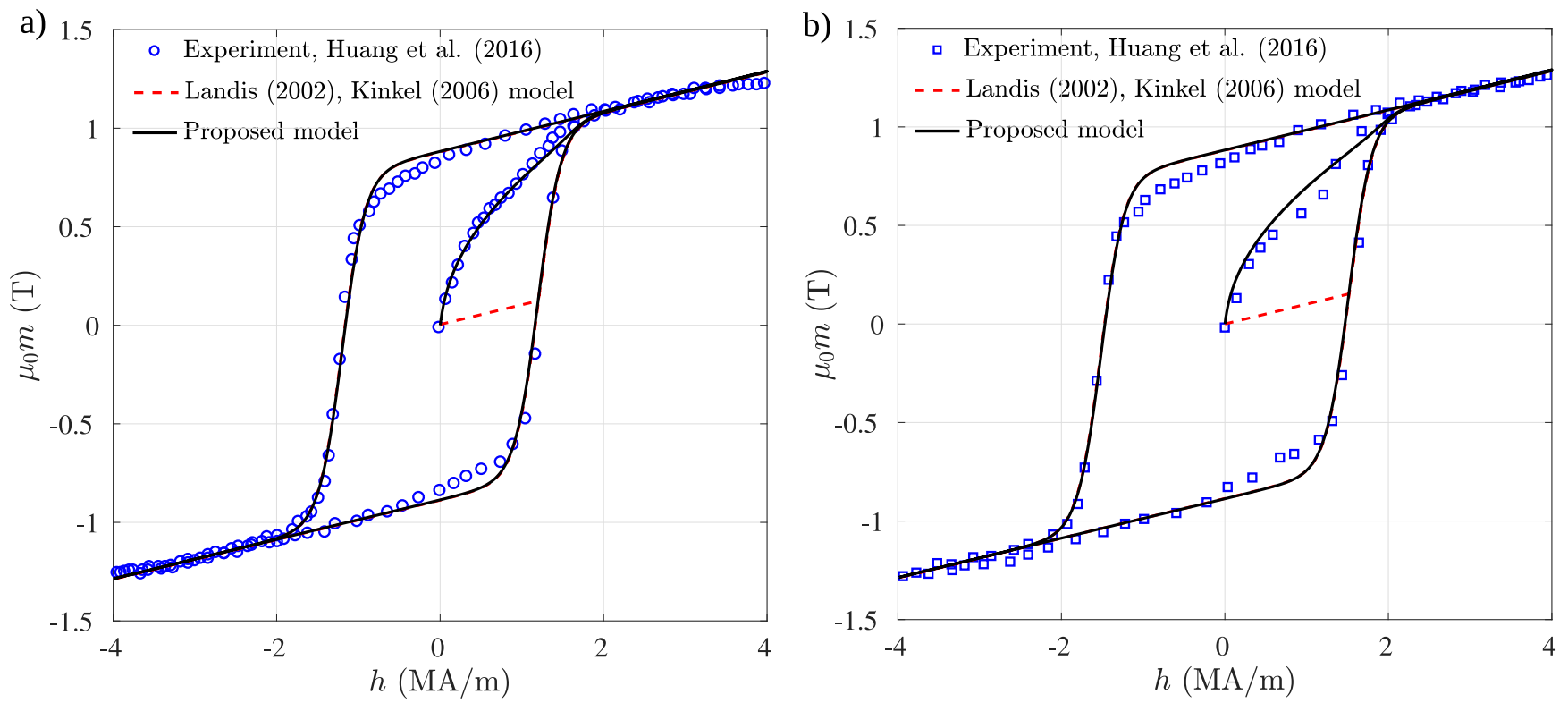

FIG. 9: Experimental m-h response of a NdFeB magnet ${ }^{6}$ with the fitted rate-independent proposed model.
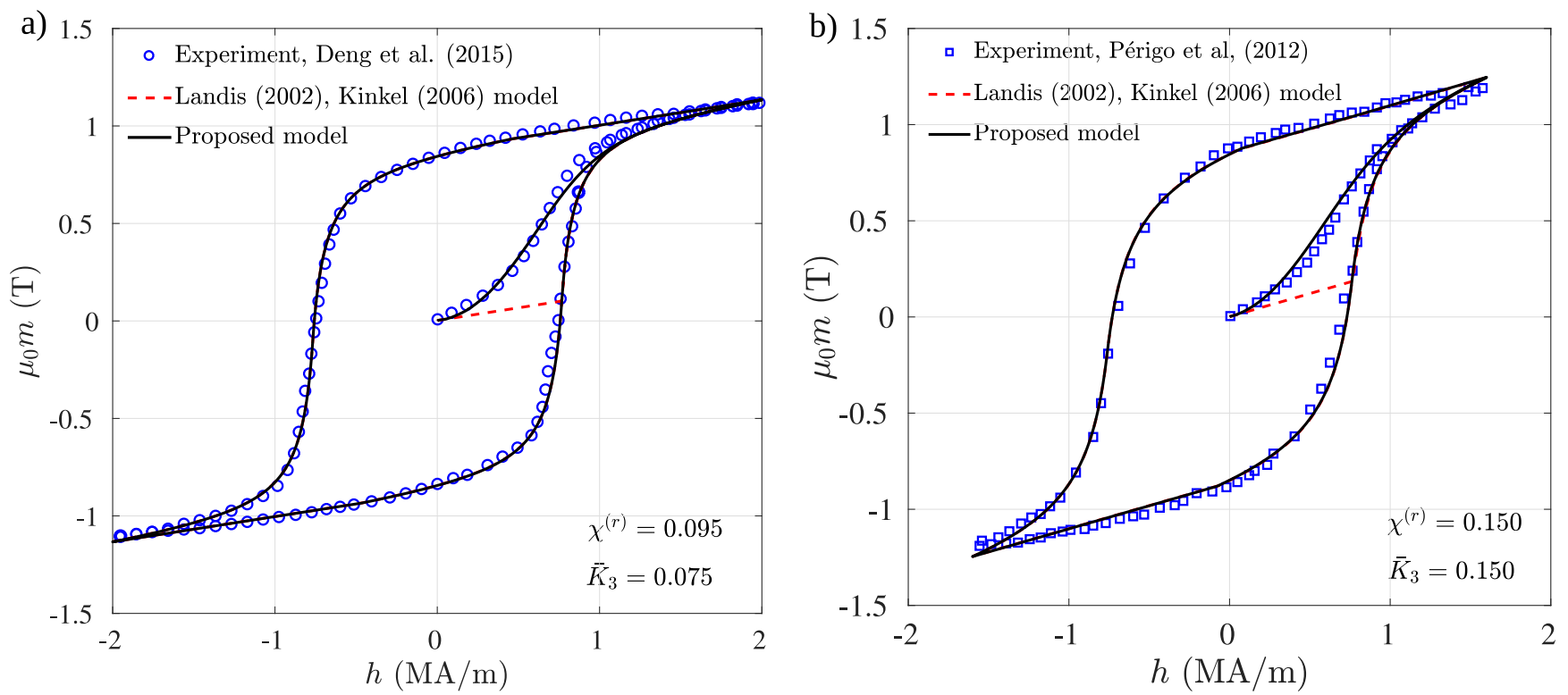

FIG. 10: Experimental m-h response of NdFeB powder from (a) Deng et al. ${ }^{5}$ and (b) Périgo et al. ${ }^{4}$ with the fitted rate-independent proposed model.

the model with one of the minor loop data to obtain $\bar{h}_{0}^{e}, k_{1}, k_{2}$ and $k_{3}$ as shown in Table III. In Fig. 11b, we use the already identified model to predict two additional experimental minor loops. We thus show that the model is able to reproduce but also predict sufficiently well the major and minor hysteresis loops of MgMn steel.

\section{Asymmetric minor loops}

In Fig. 12, we use our model to probe the experimental data for two different magnets; (a) annealed nanocrys- talline Co alloy and (b) 3\% silicon steel, subjected to uniaxial, partially-reversed loading, which results in asymmetric minor loops. The experimental $m-h$ response along with three first-order reversal curves (FORCs) for the annealed nanocrystalline Co alloy magnet is obtained by Martínez-García et al. ${ }^{62}$. Similar to the previous figures, we carry out a two step fitting of our model. First, we fit the major loop data of Martínez-García et al. ${ }^{62}$ to obtain the model parameters except $\bar{h}_{0}^{r}$ and $q$, which are inconsequential due to unavailability of the initial magnetization data. We then use the model to probe one of the experimental FORC data thus identifying the pa- 

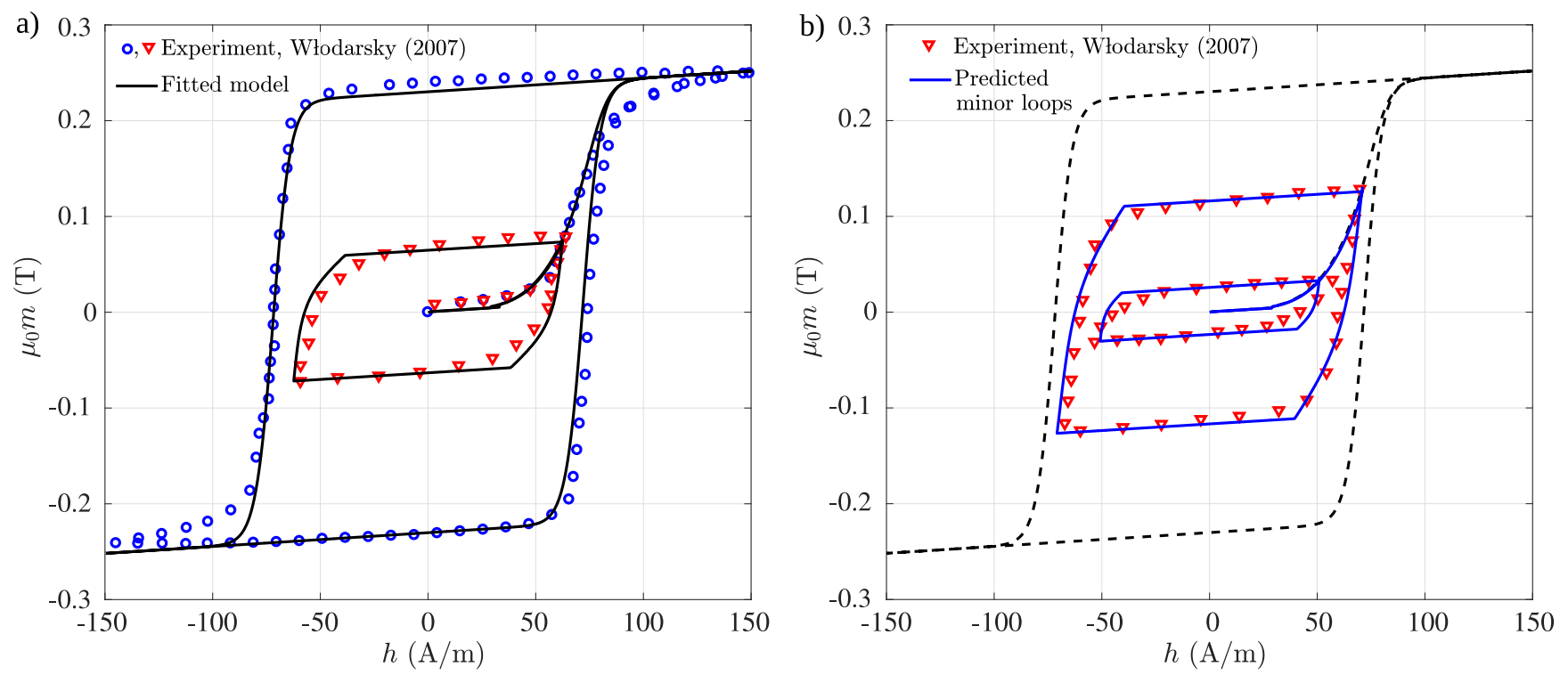

FIG. 11: Experimental m-h response of MgMn Steel by Włodarski ${ }^{39}$ with (a) the fitted model and (b) the model predictions.

rameters $\bar{h}_{0}^{e}, k_{1}, k_{2}$ and $k_{3}$. The resulting values for the model parameters are given in Table IV.

TABLE IV: Material parameters for annealed nanocrystalline Co alloy

\begin{tabular}{ll}
\hline \hline$\chi^{(r)}=0.855$ & $\bar{K}_{1}=0.718 \mathrm{kA} / \mathrm{m}$ \\
$h_{s}^{r}=51.5 \mathrm{kA} / \mathrm{m}$ & $\kappa=1.0$ \\
$\bar{b}_{c}^{\max }=9.978 \mathrm{kA} / \mathrm{m}$ & $\bar{h}_{0}^{r}=1.0 \mathrm{~A} / \mathrm{m}$ \\
$q=100.0$ & $\bar{h}_{0}^{e}=85.0 \mathrm{~A} / \mathrm{m}$ \\
$k_{1}=4.0$ & $k_{2}=1.85$ \\
$k_{3}=1.25$ & \\
\hline \hline
\end{tabular}

In Fig. 12a, the solid lines correspond to the model response that is fitted to the corresponding experimental data. The dashed lines correspond to predictions of the model without introduction of additional model parameters.

Finally, Fig. 12b shows the experimental data of Benabou et al. ${ }^{40}$ corresponding to the $b-h$ response of a $3 \%$ silicon steel sample, that is subjected to a periodic $h$-field with the superposition of third harmonics. This type of loading gives rise to asymmetric minor loops. Again, a two-step fitting procedure, consisting of fitting the major loop and only one of the minor loops from the experimental data, yields the corresponding model parameters (see Table V). Note again that the initial magnetization parameters $\bar{h}_{0}^{r}$ and $q$ remain inconsequential due to unavailability of the initial magnetization data and thus are set equal to 1 and 0 , respectively. The second minor loop is then found to be well predicted by the proposed model.

TABLE V: Material parameters for 3\% silicon steel

\begin{tabular}{ll}
$\chi^{(r)}=0.999$ & $\bar{K}_{3}=12.5 \mathrm{~A} / \mathrm{m}$ \\
$h_{s}^{r}=1000 \mathrm{~A} / \mathrm{m}$ & $\kappa=1.0$ \\
$\bar{b}_{c}^{\max }=41.0 \mathrm{~A} / \mathrm{m}$ & $\bar{h}_{0}^{r}=1.0 \mathrm{~A} / \mathrm{m}$ \\
$q=100.0$ & $\bar{h}_{0}^{e}=100.0 \mathrm{~A} / \mathrm{m}$ \\
$k_{1}=8.0$ & $k_{2}=4.0$ \\
$k_{3}=1.34$ & \\
\hline \hline
\end{tabular}

The examples presented in this section illustrate the capabilities of the model to reproduce and predict magnetic responses obtained by very complex loading histories and specifically resulting to asymmetric minor loops.

\section{FORC DIAGRAMS}

\section{CONCLUDING REMARKS}

In the present study, we propose a rate-(in)dependent constitutive model for magnetic hysteresis in terms of a nonlinear convex Helmholtz free energy and a convex dissipation potential. Qualitative connections with existing micromagnetic domain theories are discussed throughout the study. In the limit of rate independent response, the dissipation potential leads to the switching surface (similar to the yield surface in mechanical plasticity). We incorporate the effect of initial magnetic hardening of a virgin specimen by introducing an isotropic hardening law, leading to the gradual growth of the switching surface until reaching a limiting surface, which encapsulates all the internal switching surfaces during the magnetization/demagnetization. Symmetric and asymmetric minor loops are modeled by introducing a set of discrete, history-dependent thermodynamic variables, that control the shrinkage and expansion of the switching surface de- 

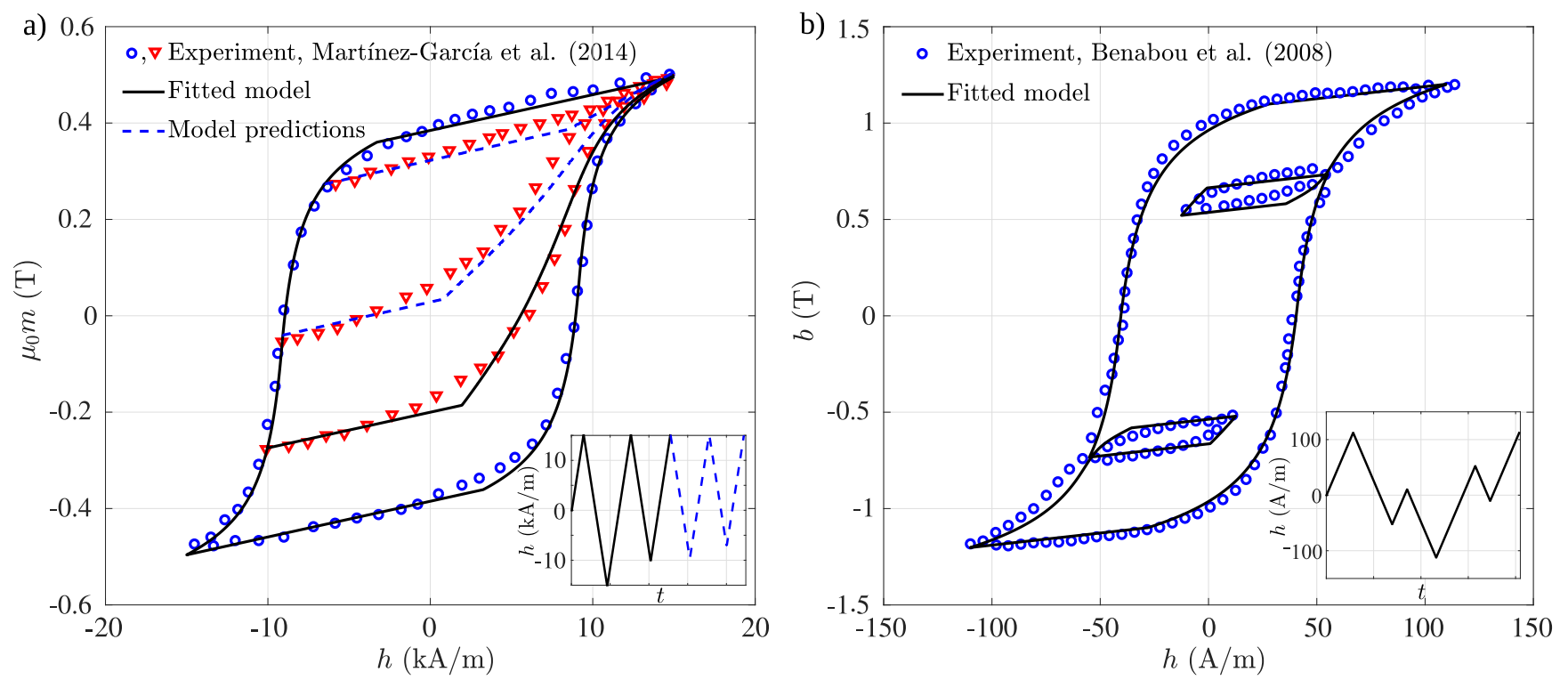

FIG. 12: (a)(inset) Loading profile for FORCs. (a) Experimental m-h response for nanocrystalline Co alloy from Martínez-García et al. ${ }^{62}$ with the model fitting and predictions. (b)(inset) Loading profile and (b) experimental b-h response for $0.3 \%$ silicon steel by Benabou et al. ${ }^{40}$ with the fitted model. Time scale is irrelevant for the rate-independent model.

pending on the loading history and the state of magnetization. In this context, we utilize the existing framework of the bounding surface (originally used in the context of mechanical plasticity) and extend it to describe the macroscopic response of permanent magnets. In particular, the present natural extension of the existing switching surface framework of McMeeking and Landis ${ }^{13}$, Landis ${ }^{14}$ and Klinkel ${ }^{15}$ enables us to model accurately the initial magnetization and the symmetric and asymmetric minor loops up to first order.

One of the key advantage of the present model is the sequential increase in the model complexity depending on the experiment we intend to model. Modeling only the major hysteresis loop does not require any isotropic hardening or shrinkage/expansion of the switching surface depending on the loading history. Hence, only the four material parameters related to the coercive field and the shape of the major loop are sufficient to describe the major hysteresis loop. On the other hand, if one needs to model also the initial magnetization response, two additional hardening parameters are further considered.

The modeling of the minor hysteresis loops necessitates a more tedious definition of the switching surface depending on the state of magnetization and the loading history, whereas it requires the determination of three additional material parameters. This sequential increase in the model complexity also allows us to probe various experimental data by identifying the model parameters in multiple independent steps. In the present work, we use at most eleven model parameters to probe the most complex experimental loading history corresponding to first order asymmetric minor loops (FOMLs). This makes the corresponding parameter identification a well-controlled and fairly straightforward task contrary to the pseudo particle model $^{31,42}$, which requires 30 to 50 parameters that need to be identified all at once.

Specifically, the model has been used to probe and predict several experimental data including the $m-h$ responses of the purely nucleation-type sintered NdFeB magnets, the combined nucleation-pinning-type $\mathrm{NdFeB}$ powder samples, the annealed nanocrystalline Co alloy and the $b-h$ responses of the $\mathrm{MnMg}$ steel and the $3 \% \mathrm{Si}$ steel. Symmetric and asymmetric minor loop data from different experiments are used to probe the effectiveness of the proposed evolution/shrinkage law for the coercive field $b_{c}$. Excellent recovery and whenever available also excellent prediction of available experiments is obtained in the present study.

The model can be extended further to model the ratedependent response of hard magnets by considering the dissipation potential to be the same power law with $0<n \ll \infty$ along with some additional terms incorporating the eddy current and the anomalous loss. Anisotropic polycrystal or single crystal magnets with a preferred direction can be modeled by a straightforward extension of the present framework, by following ideas analogous to the Hill and single-crystal mechanical plasticity. In addition, the present model is a first step towards analyzing the response of magnetorheological elastomers made of permanently magnetized particles subjected to complex magnetic and mechanical loading histories (see the very recent work of Kalina et al. ${ }^{42}$ in this direction). Finally, the proposed framework is fairly general and uses vectorial quantities thus allowing its full three dimensional implementation in numerical schemes (such as the finite elements or fast Fourier transform methods). 


\section{ACKNOWLEDGEMENTS}

This work was supported by the European Research Council (ERC) under the European Unions Horizon 2020 research and innovation program (grant agreement No 636903 - MAGNETO).

\section{APPENDIX A}

In this section, we describe the numerical integration of the proposed model. We assume that the state of magnetization is fully known at the time $t_{n}$. For an increment in the $h$-field $\Delta \mathbf{h}$, the state of the magnet at $t_{n+1}=t_{n}+\Delta t$ is sought to be determined. We employ the radial return algorithm by Ortiz and Simo ${ }^{66}$ to obtain the increment in the remanent field $\Delta \mathbf{h}^{r}$ corresponding to $\Delta \mathbf{h}$.

The determination of $\Delta \mathbf{h}^{r}$ requires defining an associated magnetic switching rule, which we obtain by first defining the total Lagrangian:

$$
\mathcal{L}=-\mathbf{b}^{r} \cdot \dot{\mathbf{h}}^{r}+\dot{\lambda} \phi\left(\mathbf{b}^{r}, \bar{h}^{r}, \xi_{N}\right),
$$

where $\dot{\lambda}$ is the Lagrange multiplier. Now, the minimization of $\mathcal{L}$ leads to the Kuhn-Tucker conditions

$$
\dot{\mathbf{h}}^{r}=\dot{\lambda} \frac{\partial \phi}{\partial \mathbf{b}^{r}}=2 \dot{\lambda} \mathbf{b}^{r},
$$

with $\dot{\lambda}=0$ for $\phi<0$ and $\dot{\lambda}>0$ for $\phi=0$, where the above represents the associated switching rule, which is equivalent to the associated flow rule in mechanical plasticity. Thus, the evolution of $\mathbf{h}^{r}$ is computed from (A.2) while $\dot{\lambda}>0$ together with the application of the constraint $\phi=0$.

We use an unconditionally stable backward Euler iteration method to solve (A.2) in time ${ }^{15,16}$. We note that during the energetic response, i.e., when $\phi\left(\mathbf{b}^{r}, b_{c}\right)<0$, the condition $\dot{\lambda}=0$ reduce (A.2) to $\dot{\mathbf{h}}^{r}=0$. Hence, $\mathbf{h}^{r}$ at $t_{n+1}$ is simply

$$
\mathbf{h}_{n+1}^{r}=\mathbf{h}_{n}^{r}
$$

Thus, we see that $\mathbf{h}^{r}$ remains constant at the time of the energetic response. During the magnetic switching, i.e. $\phi\left(\mathbf{b}^{r}, b_{c}\right)=0,(\mathrm{~A} .2)$ is expressed as

$$
\mathbf{h}_{n+1}^{r}=\mathbf{h}_{n}^{r}+2 \gamma_{n} \mathbf{b}_{n}^{r}
$$

where $\gamma_{n}=\dot{\lambda}\left(t_{n}-t_{n-1}\right)$, and the residues, corresponding to (A.2) and (19) are calculated as

$$
\mathbf{r}_{a}=\mathbf{h}_{n+1}^{r}-\mathbf{h}_{n}^{r}-2 \gamma_{n} \mathbf{b}_{n}^{r}
$$

and

$$
r_{b}=\mathbf{b}_{n+1}^{r} \cdot \mathbf{b}_{n+1}^{r}-\left\{b_{c}\left(\xi_{2 p+4(n)}\right)\right\}^{2},
$$

respectively. The above two equations together forms a $4 \times 1$ residue vector $\mathbf{R}=\left[\mathbf{r}_{a}, r_{b}\right]^{T}$. Note that, in (A.6) the switching surface radius $b_{c}$ is a function of $2 p+4$ historydependent parameters, which are stored up to the $n^{\text {th }}$ increment. Thus, the $b_{c}$ in the $n+1^{\text {th }}$ increment is obtained from the history variables, stored at the previous increment.

Now, if the residue norm $\|\mathbf{R}\|$ is greater than a prescribed tolerance value, we need to correct $\mathbf{g}_{n+1}=$ $\left[\mathbf{h}_{n+1}^{r}, \gamma_{n+1}\right]^{T}$ from a local Newton iteration, which can be accomplished by calculating $\Delta \mathbf{g}=\left[\Delta \mathbf{h}^{r}, \Delta \gamma\right]^{T}$ from the following

$$
\mathbf{G}_{n+1}^{(k)} \Delta \mathbf{g}=-\mathbf{R}^{(k)},
$$

where $\mathbf{G}_{n+1}^{(k)}=\partial \mathbf{R}^{(k)} / \partial \mathbf{g}_{n+1}$ is a $4 \times 4$ matrix. Next, the $\mathbf{g}_{n+1}$ is modified after $k^{\text {th }}$ local Newton iteration as

$$
\mathbf{g}_{n+1}^{(k+1)}=\mathbf{g}_{n+1}^{(k)}+\Delta \mathbf{g}
$$

This local iteration continues until $\|\mathbf{R}\|^{(k+1)}$ becomes less than the prescribed tolerance.

\section{APPENDIX B}

It is observed that a singularity may appear in equation $(37)_{3}$ in the degenerate case of $\delta_{(p)}^{D} \rightarrow 0$. To overcome this problem, one may augment $(37)_{2}$ with an additional term, such as

$$
\begin{aligned}
b_{c(p)} & =b_{c}^{(0)}\left\{1-k_{1}\left(1-\frac{\delta_{(p)}}{2 h_{s}^{r}}\right) \frac{R_{\mathbf{h}^{r}}}{h_{s}^{r}} \mathscr{F}_{3} \mathscr{F}_{1}\right\} \\
& +\left[b_{c}^{\max }\left\{\tanh \left(\frac{\bar{h}^{r}}{\bar{h}_{0}^{r}}\right)\right\}^{1 / q}-b_{c(0)}\right]\left(1-\mathscr{F}_{3}\right) \mathscr{F}_{4},
\end{aligned}
$$

where the functions $\mathscr{F}_{3}$ and $\mathscr{F}_{4}$ are now defined by

$$
\begin{aligned}
& \mathscr{F}_{3}=\tanh \left(6.0 \frac{\delta_{(p)}^{D}}{h_{s}^{r}}\right), \\
& \mathscr{F}_{4}=\tanh \left\{\frac{2 R_{\mathbf{h}^{r}}}{\delta_{(p)}^{D}}\left(\frac{\bar{h}^{e}}{\bar{h}_{0}^{e}}-\frac{\bar{h}_{(p)}^{e}}{\bar{h}_{0}^{e}}\right)\right\},
\end{aligned}
$$

respectively. Now, for $\delta_{(p)}^{D}=0, \mathscr{F}_{3}$ vanishes, reducing (B.1) to its second term only. Therefore, instead of decreasing, the $b_{c}$ rather increases to $b_{c}^{\max }$ during the energetic response by virtue of the saturation function $\mathscr{F}_{4}$.

${ }^{1}$ G. C. Hadjipanayis and W. Gong, Journal of Applied Physics 64, 5559 (1988).

${ }^{2}$ Z. Gao, D. C. Jiles, D. J. Branagan, and R. W. McCallum, Journal of Applied Physics 79, 5510 (1996).

${ }^{3}$ Z. Liu, H. Huang, X. Gao, H. Yu, X. Zhong, J. Zhu, and D. Zeng, Journal of Physics D: Applied Physics 44, 025003 (2010).

${ }^{4}$ E. Périgo, M. de Campos, R. Faria, and F. Landgraf, Powder technology 224, 291 (2012).

${ }^{5}$ X. Deng, Z. Liu, H. Yu, Z. Xiao, and G. Zhang, Journal of Magnetism and Magnetic Materials 390, 26 (2015). 
${ }^{6}$ Y. Huang, Y. Wang, Y. Hou, Y. Wang, Y. Wu, S. Ma, Z. Liu, D. Zeng, Y. Tian, W. Xia, et al., Journal of Magnetism and Magnetic Materials 399, 175 (2016).

${ }^{7}$ J. M. Linke, D. Y. Borin, and S. Odenbach, RSC Advances 6, 100407 (2016).

${ }^{8}$ M. Schümann, D. Borin, S. Huang, G. Auernhammer, R. Müller, and S. Odenbach, Smart Materials and Structures 26, 095018 (2017).

${ }^{9}$ Q. Wen, Y. Wang, and X. Gong, Smart Materials and Structures 26, 075012 (2017).

${ }^{10}$ P. A. Sánchez, T. Gundermann, A. Dobroserdova, S. S. Kantorovich, and S. Odenbach, Soft Matter (2018), 10.1039/c7sm02366a.

${ }^{11}$ F. N. A. L. C. M. M. R. M. Huber, J E, Journal of the Mechanics and Physics of Solids 47, 1663 (1999).

12 A. C. Cocks and R. M. Mcmeeking, Ferroelectrics 228, 219 (1999).

${ }^{13}$ R. M. McMeeking and C. M. Landis, International Journal of Engineering Science 40, 1553 (2002).

${ }^{14}$ C. M. Landis, Journal of the Mechanics and Physics of Solids 50, 127 (2002).

${ }^{15} \mathrm{~S}$. Klinkel, International Journal of Solids and Structures 43 7197 (2006).

${ }^{16}$ K. Linnemann, S. Klinkel, and W. Wagner, International Journal of Solids and Structures 46, 1149 (2009).

${ }^{17} \mathrm{C}$. Miehe, D. Rosato, and B. Kiefer, International Journal for Numerical Methods in Engineering 86, 1225 (2011).

${ }^{18}$ H. Kronmüller, K.-D. Durst, and M. Sagawa, Journal of Magnetism and Magnetic Materials 74, 291 (1988).

${ }^{19}$ H. Kronmüller, in Supermagnets, hard magnetic materials (Springer, 1991) pp. 461-498.

${ }^{20}$ K. Buschow, Reports on Progress in Physics 54, 1123 (1991).

${ }^{21}$ D. Givord, M. Rossignol, and D. Taylor, Le Journal de Physique IV 2, C3 (1992).

${ }^{22}$ J. Fidler and T. Schrefl, Journal of Applied Physics 79, 5029 (1996).

${ }^{23}$ K. Honda and S. Saitô, Physical Review 16, 495 (1920).

${ }^{24} \mathrm{H}$. Li, Y. Liang, X. Tan, H. Xu, P. Hu, and K. Ren, Materials 10, 1062 (2017).

${ }^{25}$ P. Gaunt, Philosophical Magazine B 48, 261 (1983).

${ }^{26}$ F. Preisach, Zeitschrift für physik 94, 277 (1935).

${ }^{27}$ D. Jiles and D. Atherton, Journal of magnetism and magnetic materials 61, 48 (1986).

${ }^{28}$ Q. Jiang, Journal of the Mechanics and Physics of Solids 41, 1599 (1993).

${ }^{29}$ Q. Jiang, Journal of Elasticity 34, 1 (1994).

${ }^{30}$ R. E. Logé and Z. Suo, Acta Materialia 44, 3429 (1996).

${ }^{31}$ A. Bergqvist, Physica B: Condensed Matter 233, 342 (1997).

${ }^{32}$ S. Hwang, C. Lynch, and R. McMeeking, Acta Metallurgica et Materialia 43, 2073 (1995).

${ }^{33}$ S. C. Hwang and R. M. Mcmeeking, Ferroelectrics 207, 465 (1998)

${ }^{34}$ S. C. Hwang and R. M. McMeeking, Ferroelectrics 211, 177 (1998).

${ }^{35}$ R. C. Smith, M. J. Dapino, and S. Seelecke, Journal of Applied Physics 93, 458 (2003).

${ }^{36}$ R. C. Smith, S. Seelecke, M. Dapino, and Z. Ounaies, Journal of the Mechanics and Physics of Solids 54, 46 (2006).
${ }^{37}$ J. E. Huber and N. A. Fleck, Journal of the Mechanics and Physics of Solids 49, 785 (2001).

${ }^{38} \mathrm{~K}$. Bhattacharya and G. Ravichandran, Acta Materialia 51, 5941 (2003).

${ }^{39} \mathrm{Z}$. Włodarski, Journal of magnetism and magnetic materials $\mathbf{3 0 8}$, 15 (2007).

${ }^{40}$ A. Benabou, J. Leite, S. Clénet, C. Simão, and N. Sadowski, Journal of magnetism and magnetic materials 320, e1034 (2008).

${ }^{41}$ V. François-Lavet, F. Henrotte, L. Stainier, L. Noels, and C. Geuzaine, Journal of Computational and Applied Mathematics 246, 243 (2013).

${ }^{42}$ K. A. Kalina, J. Brummund, P. Metsch, M. Kästner, D. Y. Borin, J. M. Linke, and S. Odenbach, Smart Materials and Structures 26, 105019 (2017).

${ }^{43}$ Y. F. Dafalias and E. P. Popov, Acta mechanica 21, 173 (1975).

${ }^{44}$ Y. F. Dafalias and E. P. Popov, Journal of applied mechanics 43 , 645 (1976).

${ }^{45}$ Y. F. Dafalias and E. P. Popov, Nuclear Engineering and Design 41, 293 (1977).

${ }^{46}$ J. Chaboche, K. D. Van, and G. Cordier, SMIRT-5, Division L. Berlin (1979).

${ }^{47}$ J.-L. Chaboche, International Journal of plasticity 2, 149 (1986).

${ }^{48}$ A. Dorfmann and R. Ogden, European Journal of MechanicsA/Solids 22, 497 (2003).

${ }^{49}$ A. Dorfmann and R. Ogden, Acta Mechanica 167, 13 (2004).

${ }^{50}$ A. Dorfmann and R. Ogden, Zeitschrift für angewandte Mathematik und Physik ZAMP 56, 718 (2005).

${ }^{51}$ K. Danas, Journal of the Mechanics and Physics of Solids 105, 25 (2017).

${ }^{52} \mathrm{~S}$. Kankanala and N. Triantafyllidis, Journal of the Mechanics and Physics of Solids 52, 2869 (2004).

${ }^{53}$ D. Rosato and C. Miehe, International Journal of Engineering Science 74, 162 (2014).

${ }^{54}$ B. Halphen and Q. Son Nguyen, Journal de Mécanique 14, 39 (1975).

${ }^{55}$ P. Germain, Q. S. Nguyen, and P. Suquet, Journal of Applied Mechanics 50, 1010 (1983).

${ }^{56} \mathrm{H}$. Hauser, Journal of Applied Physics 75, 2584 (1994).

${ }^{57}$ H. Hauser, Journal of Applied Physics 96, 2753 (2004).

${ }^{58}$ K. Danas, V. S. Deshpande, and N. A. Fleck, Journal of the Mechanics and Physics of Solids 60, 1605 (2012).

${ }^{59}$ A. Avakian and A. Ricoeur, Journal of Applied Physics 121, 053901 (2017).

${ }^{60}$ J. Herbst, Reviews of Modern Physics 63, 819 (1991).

${ }^{61}$ P. L. Fulmek and H. Hauser, Journal of Magnetism and Magnetic Materials 160, 35 (1996).

62 J. C. Martínez-García, M. Rivas, D. Lago-Cachón, and J. García, Journal of Physics D: Applied Physics 47, 015001 (2013).

${ }^{63}$ T. Miyamoto, H. Sakurai, H. Takabayashi, and M. Aoki, IEEE Transactions on Magnetics 25, 3907 (1989).

${ }^{64}$ J. M. Ginder, M. E. Nichols, L. D. Elie, and J. L. Tardiff, in Smart Structures and Materials 1999: Smart Materials Technologies, Vol. 3675 (International Society for Optics and Photonics, 1999) pp. 131-139.

${ }^{65}$ MATLAB, version 9.2.0.538062 (R2017a) (The MathWorks Inc., Natick, Massachusetts, 2017a).

${ }^{66} \mathrm{M}$. Ortiz and J. Simo, International Journal for Numerical Methods in Engineering 23, 353 (1986).

${ }^{67}$ J. Liu, H. Sepehri-Amin, T. Ohkubo, K. Hioki, A. Hattori, T. Schrefl, and K. Hono, Acta Materialia 61, 5387 (2013). 